\title{
Overview of Environmental and \\ Hydrogeologic Conditions near Big Lake, Alaska
}

U.S. GEOLOGICAL SURVEY

Open-File Report 95-403

Prepared in cooperation with the

FEDERAL AVIATION ADMINISTRATION

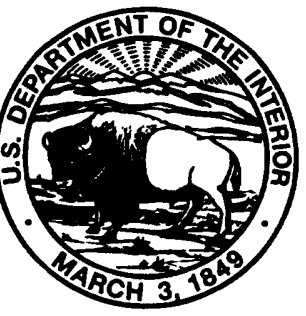




\section{Overview of Environmental and}

\section{Hydrogeologic Conditions near Big Lake, Alaska}

By Eppie V. Hogan

U.S. GEOLOGICAL SURVEY

Open-File Report 95-403

Prepared in cooperation with the

FEDERAL AVIATION ADMINISTRATION

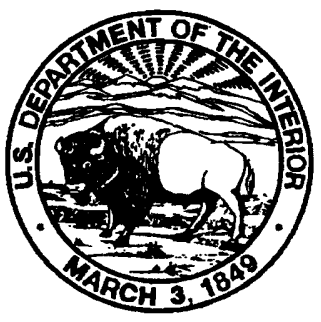

Anchorage, Alaska

1995 


\section{U.S. DEPARTMENT OF THE INTERIOR \\ BRUCE BABBITT, Secretary}

\section{U.S. GEOLOGICAL SURVEY}

Gordon P. Eaton, Director

For additional information write to:

District Chief

U.S. Geological Survey

4230 University Drive, Suite 201

Anchorage, AK 99508-4664
Copies of this report may be purchased from:

U.S. Geological Survey

Earth Science Information Center

Open-File Reports Section

Box 25286, MS 517

Federal Center

Denver, CO 80225-0425 


\section{CONTENTS}

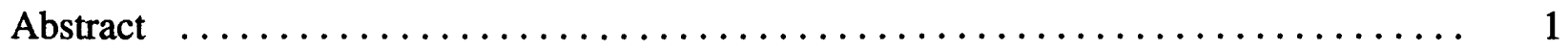

Introduction $\ldots \ldots \ldots \ldots \ldots \ldots \ldots \ldots \ldots \ldots \ldots \ldots \ldots \ldots \ldots, 2$

Background $\ldots \ldots \ldots \ldots \ldots \ldots \ldots \ldots \ldots \ldots \ldots \ldots \ldots \ldots \ldots \ldots \ldots \ldots \ldots \ldots \ldots \ldots, 2$

Location..................................... 2

Facility history $\ldots \ldots \ldots \ldots \ldots \ldots \ldots \ldots \ldots \ldots \ldots \ldots \ldots \ldots, 2$

Climate ........................................... 4

Vegetation $. . \ldots \ldots \ldots \ldots \ldots \ldots \ldots \ldots \ldots \ldots \ldots \ldots \ldots \ldots \ldots, 4$

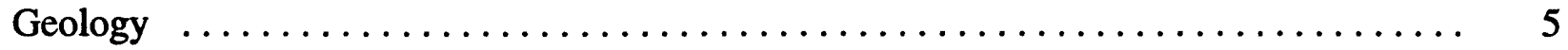

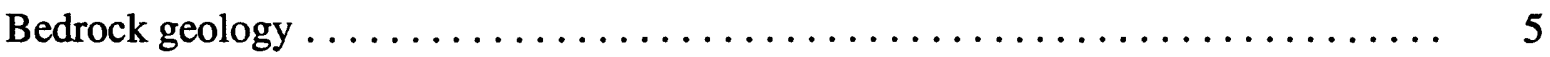

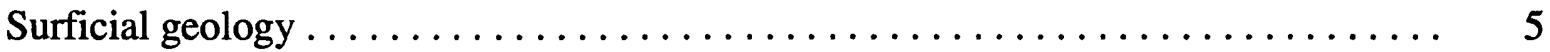

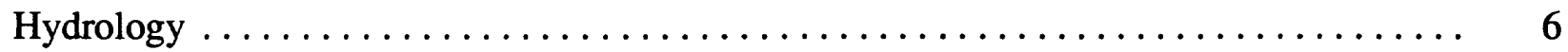

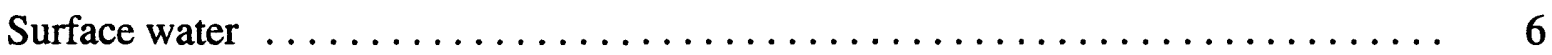

Ground water...................................... 7

Drinking water $\ldots \ldots \ldots \ldots \ldots \ldots \ldots \ldots \ldots \ldots \ldots \ldots \ldots \ldots \ldots \ldots \ldots, 8$

Summary $\ldots \ldots \ldots \ldots \ldots \ldots \ldots \ldots \ldots \ldots \ldots \ldots \ldots \ldots \ldots \ldots \ldots, \quad 9$

References cited.................................... 10

Appendix 1: Data from wells near Big Lake, Alaska $\ldots \ldots \ldots \ldots \ldots \ldots \ldots \ldots \ldots$ A-1

Appendix 2: Water-quality data near Big Lake, Alaska $\ldots \ldots \ldots \ldots \ldots \ldots \ldots \ldots$ A-2

FIGURES

1. Map showing location of Big Lake, Alaska, and the Federal Aviation

Administration facility $\ldots \ldots \ldots \ldots \ldots \ldots \ldots \ldots \ldots \ldots \ldots \ldots, 3$

\section{TABLES}

1. Mean monthly and annual temperature, precipitation, and snowfall, for the period 1957-61, Big Lake, Alaska. . .

2. Summary of limnologic characteristics of Big Lake during $1983-84 \ldots \ldots \ldots \ldots \ldots \ldots \ldots \ldots \ldots \ldots \ldots \ldots \ldots \ldots \ldots \ldots \ldots \ldots \ldots \ldots \ldots$.

3. Selected water-quality data for wells near Big Lake, Alaska ............ 9 
CONVERSION FACTORS, VERTICAL DATUM, AND ABBREVIATIONS

\begin{tabular}{lll}
\hline Multiply & By & To obtain \\
centimeter per second $(\mathrm{cm} / \mathrm{s})$ & 0.3937 & foot per second \\
millimeter $(\mathrm{mm})$ & 0.03937 & inch \\
meter $(\mathrm{m})$ & 3.281 & foot \\
square meter $\left(\mathrm{m}^{2}\right)$ & 10.76 & square foot \\
kilometer $(\mathrm{km})$ & 0.6214 & mile \\
square kilometer $\left(\mathrm{km}^{2}\right)$ & 0.3861 & square mile \\
liter per second $(\mathrm{L} / \mathrm{s})$ & 15.85 & gallon per minute \\
cubic meter $\left(\mathrm{m}^{3}\right)$ & 264.2 & gallon \\
cubic meter per second per square kilometer & 91.4 & cubic foot per second per square \\
{$\left[\left(\mathrm{m}^{3} / \mathrm{s}\right) / \mathrm{km}^{2}\right]$} & & mile \\
\hline
\end{tabular}

In this report, temperature is reported in degrees Celsius $\left({ }^{\circ} \mathrm{C}\right)$, which can be converted to degrees Fahrenheit $\left({ }^{\circ} \mathrm{F}\right)$ by the following equation:

$$
{ }^{\circ} \mathrm{F}=1.8\left({ }^{\circ} \mathrm{C}\right)+32
$$

\section{ABBREVIATED WATER-QUALITY UNITS}

Chemical concentration and water temperature are given only in metric units. Chemical concentration in water is given in milligrams per liter $(\mathrm{mg} / \mathrm{L})$ or micrograms per liter $(\mu \mathrm{g} / \mathrm{L})$. Milligrams per liter is a unit expressing the solute mass per unit volume (liter) of water. Specific conductance is given in microsiemens per centimeter $(\mu \mathrm{S} / \mathrm{cm})$ at $25^{\circ} \mathrm{C}$.

\section{VERTICAL DATUM}

Sea level: In this report, "sea level" refers to the National Geodetic Vertical Datum of 1929-A geodetic datum derived from a general adjustment of the first-order level nets of the United States and Canada, formerly called Sea Level Datum of 1929. 


\title{
Overview of Environmental and Hydrogeologic Conditions near Big Lake, Alaska
}

\author{
By Eppie V. Hogan
}

\begin{abstract}
Big Lake, an inland lake about 12 square kilometers in area, is in south-central Alaska at latitude $61^{\circ} 33^{\prime} \mathrm{N}$., and longitude $149^{\circ} 55^{\prime} \mathrm{W}$. The community of Big Lake is on the shore of Big Lake, about 30 kilometers north of Anchorage. The Federal Aviation Administration owns or operates airway support facilities near Big Lake at latitude $61^{\circ} 34^{\prime} \mathrm{N}$., and longitude $149^{\circ} 57^{\prime} \mathrm{W}$. They wish to consider environmental and hydrogeologic conditions when evaluating options for environmental compliance and remediation at these facilities. Big Lake is in a transitional climate zone where seasonal climate patterns are not sharply defined, fluctuate from year to year, and may resemble those of either the maritime or continental climate zones. The local vegetation consists of lowland spruce-hardwood forest and low brush muskeg. Little is known about the composition and structure of the bedrock around Big Lake. The Talkeetna Mountains to the northeast consist chiefly of igneous rocks including granitic rocks, lava, and tuff; the Chugach Mountains to the southeast consist of granitic intrusive rocks of Mesozoic age, metamorphosed sedimentary rocks, and greenstone; and the Alaska Range to the northwest consists of granites, volcanic' rocks, argillite, shale, sandstone, and siltstone. The principal surficial materials near Big Lake consist of outwash sand and gravel deposits, partly saturated gravel in ground moraines, and windblown sediment. Relief is low and drainage in the Big Lake area is poor; there are large areas of swampy ground and numerous lakes and ponds. The glacially derived sediments near Big Lake contain ground water in both confined and unconfined aquifers. Residents in the Big Lake area use ground water as their principal drinking-water source. Big Lake is a potential alternative drinking-water source; however, data are inadequate to characterize the present quality of Big Lake water in relation to current drinking-water standards.
\end{abstract}




\section{INTRODUCTION}

The Federal Aviation Administration (FAA) owns and (or) operates airway support and navigational facilities throughout Alaska. At many of these sites, fuels and potentially hazardous materials such as solvents, polychlorinated biphenyls, and pesticides may have been used and (or) disposed of. To determine if environmentally hazardous materials have been spilled or discarded at the sites, the FAA is conducting environmental studies mandated under the Comprehensive Environmental Response, Compensation, and Liability Act (CERCLA) and the Resource Conservation and Recovery Act (RCRA). To complete these more comprehensive environmental studies, the FAA requires information on the hydrology and geology of areas surrounding the sites. This report, the product of compilation, review, and summary of existing hydrologic and geologic data by the U.S. Geological Survey (USGS), in cooperation with the FAA, provides such information for the FAA facility and nearby areas at Big Lake, Alaska. Also presented in this report are brief descriptions of the history of the FAA facility and the physical setting of the area.

\section{BACKGROUND}

\section{Location}

Big Lake is in south-central Alaska at lat $61^{\circ} 33^{\prime} \mathrm{N}$., long $149^{\circ} 55^{\prime} \mathrm{W}$. The community of Big Lake is on the shore of Big Lake, about $30 \mathrm{~km}$ north of Anchorage (fig. 1). The area is part of the Upper Matanuska-Susitna Valley, a glacial trough containing longitudinal bedrock hills and small narrow lakes (Wahrhaftig, 1965). The Big Lake FAA facility is at lat $61^{\circ} 34^{\prime} \mathrm{N}$., long $149^{\circ} 57^{\prime} \mathrm{W}$, about $3 \mathrm{~km}$ northwest of Big Lake and about $1.5 \mathrm{~km}$ west of Horseshoe Lake (fig. 1).

\section{Facility History}

The FAA involvement near Big Lake began in 1962 when land was leased from the Alaska Department of Natural Resources to construct a Very High Frequency Omnidirectional Range Station/Tactical Air Communication facility (VORTAC). The VORTAC facility is maintained by personnel stationed in Anchorage and is the only FAA facility at Big Lake. A more detailed description of the VORTAC facility at Big Lake and a list of suspected sources of environmental contamination is given in an environmental compliance investigation report by Ecology and Environment Inc., (1993). 


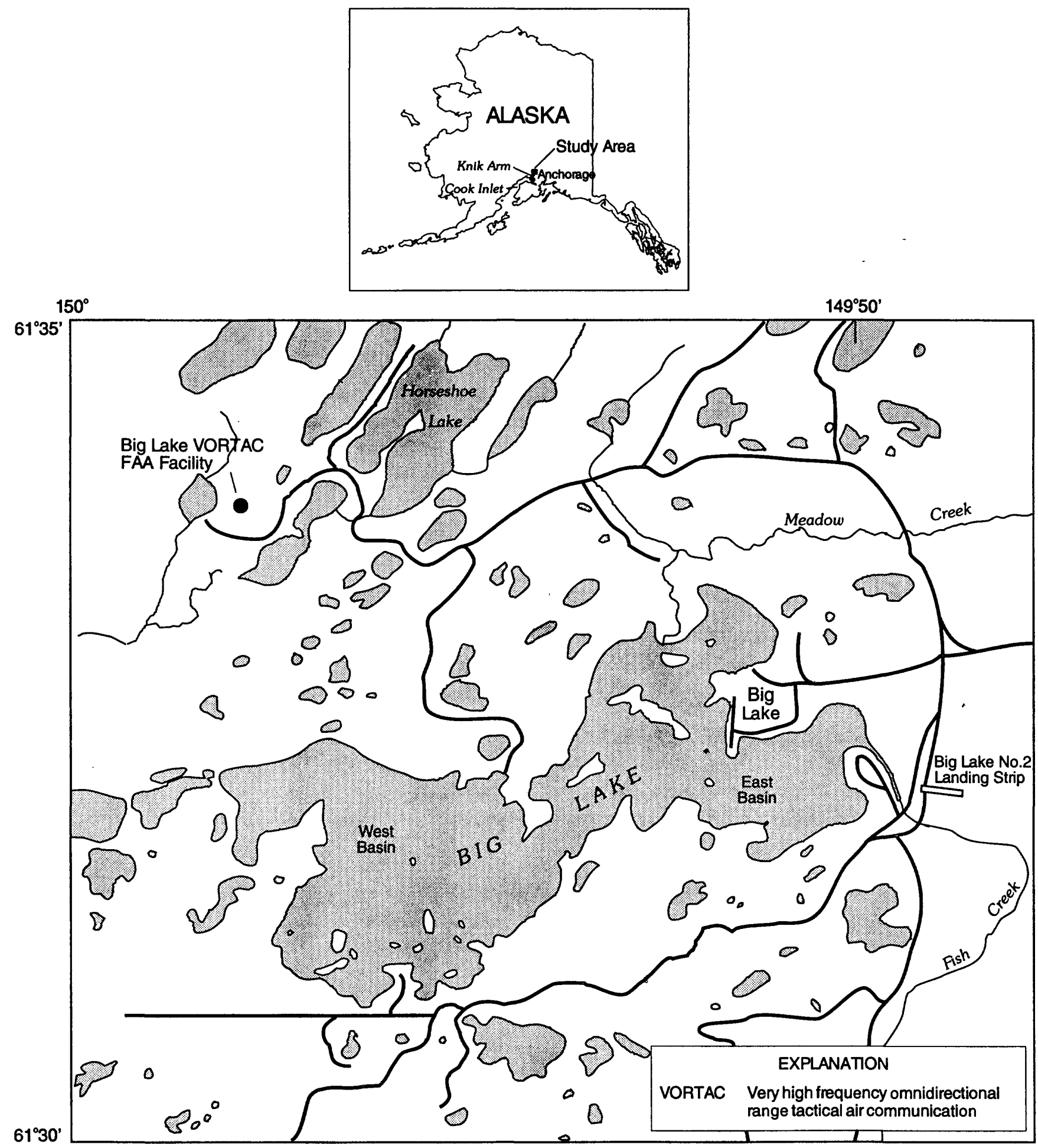

Base from U.S.Geological Survey, Anchorage (C-8), Alaska, 1:63,360, 1950.

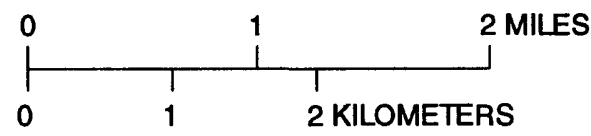

Figure 1. Location of Big Lake and the Federal Aviation Administration facility. 


\section{Climate}

Big Lake is in a transitional climate zone. Seasonal climate patterns in this zone are not sharply defined, fluctuate from year to year, and may resemble those of either the maritime or continental climate zones (Hartman and Johnson, 1984). Big Lake has a mean annual temperature of $0.6^{\circ} \mathrm{C}$, but temperatures range from a June mean maximum of $21.1^{\circ} \mathrm{C}$ to a March mean minimum of $-19.7^{\circ} \mathrm{C}$ (Leslie, 1989; table 1 this report). Mean annual precipitation is about 565 $\mathrm{mm}$ and mean annual snowfall is about $1,490 \mathrm{~mm}$. Values for mean monthly and annual temperature, precipitation, and snowfall for the period 1957 to 1961 for Big Lake, Alaska, are given in table 1 .

Table 1. Mean monthly and annual temperature, precipitation, and snowfall, for the period 1957-61, Big Lake, Alaska

[Modified from Leslie, $1989 ;{ }^{\circ} \mathrm{C}$, degree Celsius; mm, millimeter]

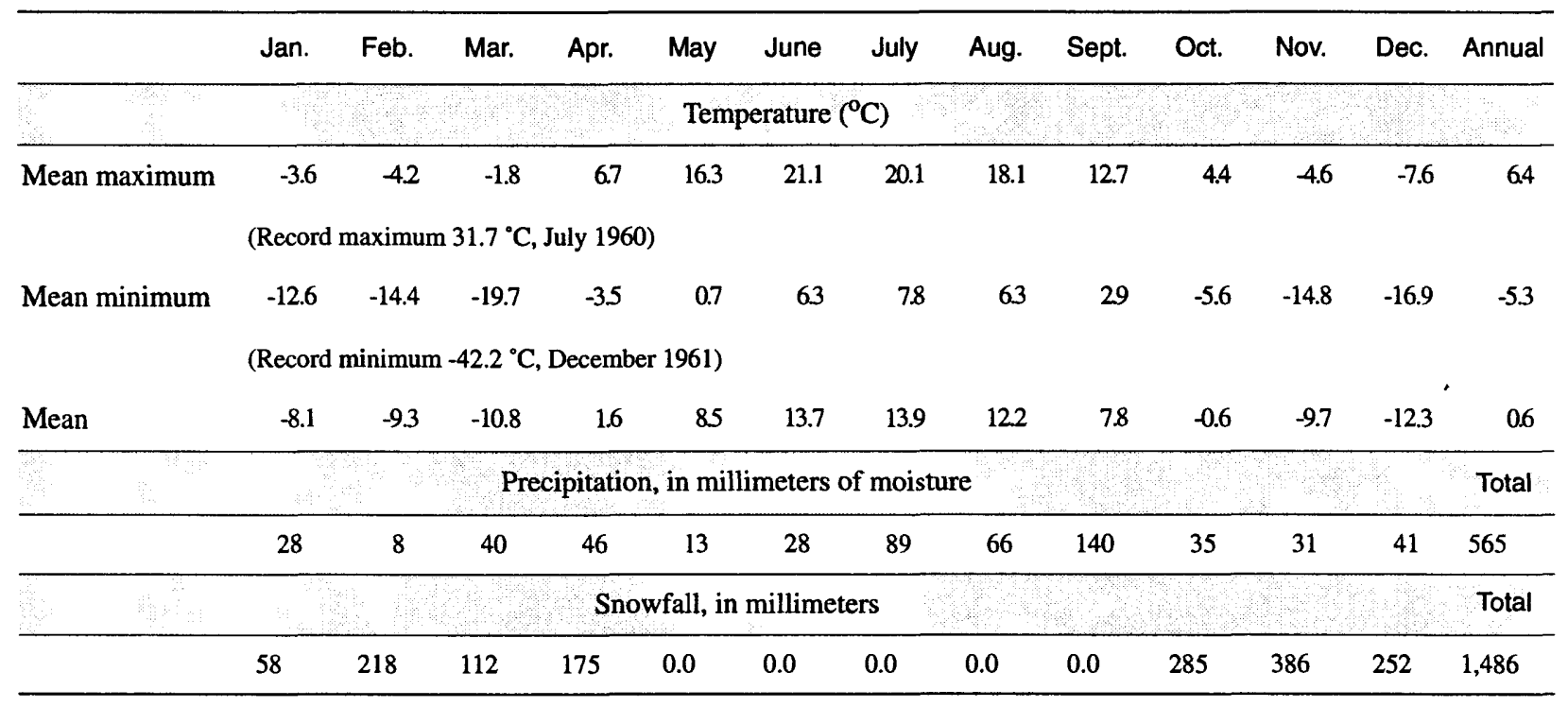

\section{Vegetation}

The vegetation surrounding Big Lake consists of lowland spruce-hardwood forest and low brush muskeg (Viereck and Little, 1972; Selkregg, 1976). The forested areas typically occur on shallow peat deposits, glacial deposits, and outwash plains and consist of evergreen and deciduous trees, including stands of black spruce. Undergrowth in the forested areas consists of willows and other brush (Selkregg, 1976). Dwarf shrub vegetation dominates areas of low brush muskeg. Western hemlock, Alaska cedar, sedges, mosses, and lichens are also found in these areas (Selkregg, 1976). 


\section{GEOLOGY}

\section{Bedrock Geology}

The bedrock of the Matanuska-Susitna Valley is described by Trainer (1960) and Freethey and Scully (1980). The Talkeetna Mountains, about $20 \mathrm{~km}$ to the northeast, consist of igneous rocks including granitic rocks, lava, and tuff. Sedimentary rocks of Cretaceous and Tertiary age form the south flank of the mountains (Trainer, 1960). The Chugach Mountains, about $50 \mathrm{~km}$ to the southeast, consist of Mesozoic granitic intrusive rocks, metamorphosed sedimentary rocks-mainly slate, argillite, and graywacke — and greenstone (Trainer, 1960). The Alaska Range, about $75 \mathrm{~km}$ to the northwest, consists of granites, volcanic rocks, argillite, shale, sandstone, and siltstone (Freethey and Scully, 1980). Sedimentary rocks consisting of sandstone, shale, and coal of Cretaceous age extend down the Matanuska Valley to Moose Creek, about $45 \mathrm{~km}$ northeast of Big Lake (Trainer, 1960). Little is known about the composition and structure of the bedrock around Big Lake. Wells drilled to depths greater than $60 \mathrm{~m}$ terminated in unconsolidated materials, which may be more than $300 \mathrm{~m}$ thick in the Big Lake area (appendix 1; Freethey and Scully, 1980).

\section{Surficial Geology}

Unconsolidated materials of Quaternary age extend over most of the Matanuska-Susitna Valley floor and are predominately till, glacial outwash, and glacial-lacustrine deposits (Trainer, 1960; Freethey and Scully, 1980). The principal surficial deposits near Big Lake are outwash sand and gravel, partly saturated gravel in ground moraines, and windblown sediment (Trainer, 1960).

Outwash deposits are predominately sand, pebbly sand, gravel, and silt. Information from well logs indicates that individual layers range from a meter to as much as $70 \mathrm{~m}$ in thickness (Trainer, 1960; Freethey and Scully, 1980). The bedding is moderately well developed and crossbedding is common (Trainer, 1960).

Till is the principal component of the end moraine near Big Lake, which marks the westernmost extent of glacial advance (Trainer, 1960). Till in the Big Lake area consists of subangular to rounded rock fragments in a matrix of sand, silt, and clay. The rock fragments are derived from adjacent mountains and range in size from sand to boulders (Trainer, 1960). Because of its clay composition, the end moraine likely forms a shallow ground-water divide.

Glacial-lacustrine deposits, consisting of silt or sandy silt (loess), cover the land surface everywhere in the Matanuska-Susitna Valley except on modern flood plains, tidal flats, and steep bedrock slopes (Trainer, 1960). These sediments are relatively impermeable and are the parent materials for most of the soils in the valley. The Big Lake area is typically free of permafrost (Ferrians, 1965). However, in lowland areas where surface insulation is high and incident solar radiation is low, isolated patches of permafrost are as thick as $9 \mathrm{~m}$ and range in area up to $50 \mathrm{~m}^{2}$. 


\section{HYDROLOGY}

\section{Surface Water}

Relief is low and drainage is poor near Big Lake and there are large areas of swampy ground and numerous lakes and ponds. Mean annual runoff in the area is about $0.005\left(\mathrm{~m}^{3} / \mathrm{s}\right) / \mathrm{km}^{2}$ (Freethey and Scully, 1980). Big Lake and Horseshoe Lake are the most significant surface-water bodies near the FAA VORTAC facility (fig. 1). Big Lake covers an area of about $12 \mathrm{~km}^{2}$ and is located about $3 \mathrm{~km}$ southeast of the facility. Horseshoe Lake covers an area of about $3.5 \mathrm{~km}^{2}$ and is about $1.5 \mathrm{~km}$ east of the facility.

Big Lake consists of an east and a west basin connected by a constriction near the middle of the lake (Woods, 1992; fig. 1). The lake contains 22 islands and the shoreline length, excluding islands, is about $27 \mathrm{~km}$. The surface elevation of the lake is about $44 \mathrm{~m}$ and it has a mean depth of about $9 \mathrm{~m}$ (Woods, 1992). Meadow Creek is the major inlet stream and Fish Creek is the lake outlet (fig. 1). The VORTAC facility is not within the drainage basins of Big Lake and Horseshoe Lake. Contaminants spilled or disposed at the VORTAC facility, therefore, are unlikely to reach these surface-water bodies by surface drainage. However, they may be hydraulically connected to the shallow ground-water system.

During 1983-84, the USGS conducted a limnological study to evaluate potential eutrophication of Big Lake (Woods, 1992). Eutrophication is the enrichment of waters by nutrients, typically nitrogen and phosphorus. An excess of nutrients, generally the result of human activities, may result in increases in oxygen demand and primary production and a decrease in overall water quality. The Big Lake study involved describing and interpreting spatial and temporal variations in numerous physical, chemical, and biological characteristics. The results of the study are summarized in table 2 . The lake was classified as oligotrophic, a condition characterized by 1) a low dissolved-nutrient concentration, 2) sparse, yet diverse plant and animal life, and 3) a high dissolved-oxygen concentration. However, upon summer stratification and under ice cover, the lake's bottom waters are rapidly depleted of oxygen (Woods, 1992). Consequently, if the oxygen demand increases, the lake is susceptible to eutrophication. Similar hydrologic data for Horseshoe Lake were not found. 
Table 2. Summary of limnologic characteristics of Big Lake during 1983-84

[Modified from Woods, 1992; mg/L, milligrams per liter; $\mu \mathrm{g} / \mathrm{L}$, micrograms per liter; <, less than]

During May through October, the lake received 76 percent of the annual input of solar irradiance.

The maximum water temperature measured was $18.7^{\circ} \mathrm{C}$.

The lake was dimictic (stratifies in the summer and winter) and circulated in May and October.

Thermal stratification was well developed and persistent from June through September.

The spring circulation failed to completely reaerate the hypolimnion ${ }^{1}$.

Water was of calcium bicarbonate type.

Specific conductance ranged from 85 to $161 \mu \mathrm{S} / \mathrm{cm}$ at (25 degrees Celsius).

$\mathrm{pH}$ ranged from 6.2 to 8.0.

Dissolved-oxygen concentrations ranged from 0 to $14.7 \mathrm{mg} / \mathrm{L}$; percent saturation ranged from 0 to 124 .

Total ammonia plus organic nitrogen ranged from 110 to $940 \mu \mathrm{g} / \mathrm{L}$.

Dissolved-ammonia concentrations ranged from $<1$ to $502 \mu \mathrm{g} / \mathrm{L}$.

Dissolved-nitrite plus nitrate concentrations ranged from $<1$ to $192 \mu \mathrm{g} / \mathrm{L}$.

Total phosphorous ranged from 6 to $173 \mu \mathrm{g} / \mathrm{L}$.

Dissolved orthophosphate ranged from 1 to $66 \mu \mathrm{g} / \mathrm{L}$.

Nitrogen was the nutrient most likely to limit phytoplankton growth during the summer.

Chlorophyll- $a$ averaged $2.5 \mu \mathrm{g} / \mathrm{L}$ but peaked at $46.5 \mu \mathrm{g} / \mathrm{L}$.

Annual primary production was 29.6 grams of carbon $/ \mathrm{m}^{2}$; about 90 percent was produced during May-October.

Big Lake was oligotrophic ${ }^{2}$ during 1983-84.

${ }^{1}$ The hypolimnion is the lower stratum of a lake. It is typically colder in temperature than upper layers and is relatively undisturbed.

${ }^{2}$ An oligotrophic lake is characterized by a low dissolved-nutrient concentration, sparse yet diverse aquatic life, and a high dissolved-oxygen concentration.

\section{Ground Water}

The glacially derived sediments near Big Lake contain ground water in both confined and unconfined aquifers (Trainer, 1960; Freethey and Scully, 1980). The principal aquifers near Big Lake are composed of outwash sand and gravel laid down by glacial meltwater streams (Trainer, 1960; Freethey and Scully, 1980). The till and the bedrock are aquifers of minor importance. Because till layers contain fine-grained materials such as silt and clay, they may act as confining beds. Till generally has low permeability, although locally thin layers of sandy material may transmit small quantities of water (Trainer, 1960). The bedrock is considered impermeable and yields water only from fractures, the location and frequency of which are unknown in the Big Lake area. Several wells in bedrock in other areas of the Matanuska-Susitna Valley have obtained saltwater that is thought to have been in the rock since the region was last covered by marine water (Trainer, 1960). 
Numerous wells have been drilled in the Big Lake-Horseshoe Lake area (appendix 1). Most of the wells penetrate water-bearing sand and gravel outwash deposits that have hydraulic conductivities between $5 \times 10^{-5}$ and $10^{-6} \mathrm{~cm} / \mathrm{s}$ (Arctic Engineers Inc., 1982). These outwash deposits are of two principal forms. The first consists of 1- to 30-m-deep sheetlike deposits that lie just beneath the land surface (Trainer, 1960; Freethey and Scully, 1980; Dearborn and Allely, 1983). The water in these deposits is unconfined and overlies layers of till. The other outwash deposits are buried beneath till and may be as thick as $20 \mathrm{~m}$. These deposits commonly contain confined, or artesian, ground water (Trainer, 1960; Freethey and Scully, 1980). The aquifers vary in thickness and grainsize composition and are laterally discontinuous (Trainer, 1960). The unconfined-aquifer typically yields water at a rate of 0.3 to $3 \mathrm{~L} / \mathrm{s}$, and the confined aquifer may yield up to $18 \mathrm{~L} / \mathrm{s}$ (Trainer, 1960; Freethey and Scully, 1980; Feulner, 1968; Glass, 1983).

Ground-water recharge near Big Lake is from several sources (Freethey and Scully, 1980). Shallow aquifers are recharged primarily by infiltration of streams, lakes, and precipitation. The deeper aquifers are recharged from adjacent aquifers by leakage through the confining layers. Some confined aquifers may receive additional recharge from upgradient areas where they are exposed to surface-water sources. Ground-water levels in the area generally fluctuate less than $1 \mathrm{~m}$ throughout the year (Glass, 1983). On a regional scale, ground-water flow is south-southwest from more upland areas toward Big Lake, Fish Creek, and, ultimately, Knik Arm.

Near Big Lake, the freshwater reservoir, from the ground surface to the saltwater-freshwater interface, is about $300 \mathrm{~m}$ thick (McGee, 1977). Salinity increases with depth, and the estimated sub-sea depth to water having salt concentrations greater than $16,800 \mathrm{mg} / \mathrm{L}$ sodium chloride is about $1,800 \mathrm{~m}$.

\section{DRINKING WATER}

Residents in the Big Lake area use ground water as their principal drinking-water source. Most of the wells reach the unconfined aquifer at depths less than $10 \mathrm{~m}$ below the land surface (appendix 1; Freethey and Scully, 1980). Several wells deeper than $30 \mathrm{~m}$ reach the confined aquifer and reportedly flow at the surface (appendix 1).

In general, the ground water in the Matanuska-Susitna Valley is of adequate quality for domestic use (Trainer, 1960; Feulner, 1968; Bradley, 1976; Glass, 1983). During the last 40 years, water samples have been collected from till, outwash sand and gravel, windblown sediment, and bedrock (appendix 2). Ground water typically meets current drinking-water regulations (table 3 and appendix 2; U.S. Environmental Protection Agency, 1995). However, in some samples, concentrations of iron, manganese, chloride, and total dissolved solids exceed the recommended standard (appendix 2). Dissolved-solids concentrations range from about 50 to $200 \mathrm{mg} / \mathrm{L}$ in water from wells completed in the unconfined aquifer and from 150 to $1,400 \mathrm{mg} / \mathrm{L}$ in wells completed in the confined aquifer (Glass, 1983). In both the confined and unconfined aquifers, iron concentrations range from 0 to $7.2 \mathrm{mg} / \mathrm{L}$, chloride concentrations range from 0 to $700 \mathrm{mg} / \mathrm{L}$, and sulfate concentrations range from 0 to $130 \mathrm{mg} / \mathrm{L}$ (table 3). Big Lake contains about 100 million $\mathrm{m}^{3}$ of water and could possibly be used as an alternative drinking-water source for nearby residents; however, data are inadequate to characterize the present quality of Big Lake water in relation to all current drinking-water standards. 
Table 3. Selected water-quality data for wells near Big Lake, Alaska

[U.S. Environmental Protection Agency, 1995 and appendix 2; mg/L, milligrams per liter]

\begin{tabular}{lcc}
\hline \multicolumn{1}{c}{ Constituent (or property) } & $\begin{array}{c}\text { USEPA } \\
\text { drinking-water regulation } \\
(\mathrm{mg} / \mathrm{L})\end{array}$ & $\begin{array}{c}\text { Ground-water, } \\
\text { range in concentration } \\
(\mathrm{mg} / \mathrm{L})\end{array}$ \\
\hline Total dissolved solids & 500 & $54-1,430$ \\
Iron (Fe) & 0.3 & $0-7.2$ \\
Chloride (Cl) & 250 & $0-700$ \\
Sulfate $\left(\mathrm{SO}_{4}\right)$ & 250 & $0-130$ \\
Manganese $(\mathrm{Mn})$ & .05 & $.01-.46$ \\
Fluoride $(\mathrm{F})$ & 2 & $0-.5$ \\
$\mathrm{pH}$ (units) & $6.5-8.5$ & $6.1-8.7$ \\
\hline
\end{tabular}

\section{SUMMARY}

Big Lake is in south-central Alaska at lat $61^{\circ} 33^{\prime} \mathrm{N}$., long $149^{\circ} 42^{\prime} \mathrm{W}$. The community of Big Lake is on the shore of Big Lake, about $30 \mathrm{~km}$ north of Anchorage. The FAA operates a VORTAC facility near Big Lake. The area is in a transitional climate zone, characterized by climate patterns that are not sharply defined, fluctuate from year to year, and may resemble those of either the maritime or continental climate zones. The local vegetation consists of lowland spruce-hardwood forest and low brush muskeg. Little is known about the composition and structure of the bedrock around Big Lake. The Talkeetna Mountains to the northeast consist chiefly of igneous rocks including granitic rocks, lava, and tuff; the Chugach Mountains to the southeast consist of Mesozoic granitic intrusive rocks, metamorphosed sedimentary rocks, and greenstone; and the Alaska Range to the northwest consists of granites, volcanic rocks, argillite, shale, sandstone, and siltstone. Cretaceous sedimentary rocks consisting of sandstone, shale, and coal extend down the Matanuska-Susitna Valley to Moose Creek. The principal surficial materials near Big Lake are outwash sand and gravel deposits, partly saturated gravel in ground moraines, and windblown sediment. Drainage in the Big Lake area is poor, and there are large areas of swampy ground and numerous lakes and ponds. The glacially derived sediments near Big Lake contain ground water in both confined and unconfined aquifers. Local residents use ground water as their principal drinking-water source. Big Lake is a potential alternative drinking-water source; however, data are inadequate to characterize the present quality of Big Lake water in relation to current drinkingwater standards. 


\section{REFERENCES CITED}

Arctic Engineers, Inc., 1982, Big Lake landfill development study and plan for the Matanuska-Susitna Borough: Report 81-008-01 MSB, variously paged.

Balding, G.O., 1976, Water availability, quality, and use in Alaska: U.S. Geological Survey Open-File Report 76-513, $236 \mathrm{p}$.

Dearborn, L.L., and Allely, R.D., 1983, Water-well data for the Big Lake area, Anchorage C-8 SW quadrangle, Alaska: Alaska Division of Geological and Geophysical Surveys Report of Investigations 83-19, 1 sheet.

Ecology and Environment, Inc., 1993, Environmental Compliance Investigation Report (ECIR), Big Lake FAA station, Big Lake, Alaska: Anchorage, variously paged. [Copy available from the Environmental Compliance Section, AAL-465, Federal Aviation Administration, Alaskan Regional Office, Anchorage, Alaska].

Ferrians, O.J., Jr., comp., 1965, Permafrost map of Alaska: U.S. Geological Survey Miscellaneous Geologic Investigations Map I-445, scale 1:2,500,000.

Feulner, A.J., 1968, Data on wells in the Matanuska-Susitna Borough Area, Alaska: U.S. Geological Open-File Report, $25 \mathrm{p}$.

Freethey, G.W., and Scully, D.R., 1980, Water resources of the Cook Inlet Basin: U.S. Geological Survey Hydrologic Investigations Atlas HA-620, 4 sheets, scale 1:1,000,000.

Glass, R.L., 1983, Hydrologic data for Point MacKenzie area, southcentral Alaska: U.S. Geological Survey Open-File Report 83-142, 34 p.

Hartman, C.W., and Johnson, P.R., 1984, Environmental atlas of Alaska: University of Alaska Fairbanks, Institute of Water Resources/Engineering Experiment Station, 95 p.

Leslie, L.D., 1989, Alaska climate summaries (2d ed.): University of Alaska Anchorage, Arctic Environmental Information and Data Center, Alaska Climate Center Technical Note 5, 478 p.

McGee, D.L., 1977, Salinity study, Cook Inlet Basin, Alaska: State of Alaska, Geological and Geophysical Surveys Geological Report 54, 6 p.

Selkregg, Lydia, 1976, Alaska regional profiles-Southwest region: University of Alaska, Arctic Environmental Information and Data Center, 313 p.

Trainer, F.W., 1960, Geology and ground-water resources of the Matanuska Valley agricultural area, Alaska: U.S. Geological Survey Water-Supply Paper 1494, 116 p.

U.S. Environmental Protection Agency (USEPA), 1995, Drinking water regulations and health advisories: U.S. Environmental Protection Agency report, $10 \mathrm{p}$.

Viereck, L.A., and Little, E.L. Jr., 1972, Alaska trees and shrubs: U.S. Department of Agriculture Handbook No. 410, $265 \mathrm{p}$.

Wahrhaftig, Clyde, 1965, Physiographic divisions of Alaska: U.S. Geological Survey Professional Paper 482, 52 p.

Woods, P.F., 1992, Limnology of Big Lake, South-central Alaska, 1983-84: U.S. Geological Survey Water-Supply Paper 2382, $108 \mathrm{p}$. 


\section{APPENDIX 1}

Data from wells near Big Lake, Alaska 
BIG LAKE - 3 MILE RADIUS

LOCAL WELL NUMBER

SB01600305ABAD1 001 SB01600402ABCB1 001 SB01700316CDCA1 002 SB01700319DCAB1 004

SB01700319DCAC1 002 SB01700319DCDA1 003 SB01700319DDBC1 001 SB01700319DDBC2 001 SB01700320ACDD1 006

SB01700320CADA1 001 SB01700320CCCC1 005 SB01700320CDAC1 003 SB01700320CDAC2 003

SB01700320CDAC3 003 SB01700320CDDB1 002 SB01700320CDDB1002 20376

SB01700320CDDB2 002 SB01700320CDDB3 002

SB01700320DDDA1 004 SB01700320DDDA2 004 SB01700321ABDA1 005 SB01700321ACDA1 008

SB01700321ADCB1 004 SB01700321CDCA1 002

SB01700321CDDC1 003 SB01700328AABB1 009

SB01700328ABAA1 012

SB01700328ABBC1 014

SB01700328ABDB1 008

SB01700328ACAA1 007 SB01700328ACBC1 002

SB01700328ACCB1 006 SB01700328ADAC1 013

SB01700328ADAC1 013

SB01700328BABA1 004 SB01700328BACD1 010

SB01700328BCDC1 001

SB01700328BDBB1 005 SB01700328DBBB1 003 SB01700329AAAB1 001 SB01700329AABA1 009 SB01700329ABAD1 005

SB01700329ABBA1 019

SB01700329ABBB1 010 SB01700329ABDB1 007 SB01700329BAAA1 014

$\begin{array}{cccc}\text { PRIMARY } & & & \\ \text { USE } & \text { DEPTH } & \text { WATER } & \text { DATE } \\ \text { OF } & \text { OF WELL } & \text { LEVEL } & \text { WELL } \\ \text { WATER } & \text { (FEET) } & \text { (FEET) } & \text { CONSTRUCTED }\end{array}$

OWNER

$\begin{array}{cl}10-22-60 & \text { UNION OIL BIG LAKE } \\ 10-06-60 & \text { UNION OIL } \\ -- & \text { NERLAND JERRY } \\ 03-29-83 & \text { DUCLOS PAUL\&SARA }\end{array}$

$\begin{array}{ll}26.0 & 3.00 \\ 31.0 & 4.00\end{array}$

74.

$--$

65.0

28.0

3.00

13.00

$33.0 \quad 3.00$

$6.00 \quad 05-25-73$

37.0

37.0

37

108

30.0

-.

--

$-$

5.00

31.0

--

47.0

$--$

14.0

$\begin{array}{ll}14.0 & \\ 65.4 & 17 .\end{array}$

36.0 -.$$
\text { H }
$$

$\mathrm{H}$
$\mathrm{P}$

H

H

H

$$
53 .
$$

$--$

8.0

160

$--$

$-20.00$

75.

$--$

5.

74.0

15.00

50.

16.

16.0

--

75.

20 .

75.

20 .

29.0

82.3

$--$

14.0

$-2.00$

$05-\overline{24}-85$

99.

14.0

22.00

$60.0 \quad 41.00 \quad 01-01-64$

$30.0 \quad 8.00 \quad 01-01-71$

$89.6 \quad--$

61.

74.0

7.

15.00
$03-21-73$

$03-26-73$

$12-12-73$

$03-29-73$

$09-19-77$

05-01-71

01-11-74

02-11-71

$05-31-73$

$01-01-57$

$11-\overline{0} 1-66$

$11-01-66$
$09-10-76$

01-01-66

$01-01-63$

$06--85$

01-01-60

02-16-76

01-01-50

$05-31-78$

$10-25-83$

11-03-84

$06-30-75$

--

11-01-85

11-01-85

01-01-61

$10,-25-79$

$$
--
$$

05-05-75

07-01-67

07-14-67

06-03-77

ERICKSON EDWIN

PEDERSON HARRY

DAHLBERG OSCAR

DALHBERG IS LODGE

STRINGFELLOW SCOTT

AK DIV PKS BIG LK 1

RODRIQUEZ

RAINEY CARROL

GALLANT FRED

SILL ART

SHIP AHOY

KETCHUMS RESORT

FIEDLER WILTON $R$

BIG LAKE SUMMR CP

I SLAND LODGE

ROCCA CLAYTON

VREM TRACY\&LINDA

BIG Y GROCERY

KOWALSKI ALVES\&BETTY

HERMANS PLACE

BIG LAKE ELEM MAT-SU

PUNCHES LAWRENCE\&FLO

STALLONE JOHN\&BARBAR

WINCHESTER MIKE

BIG LAKE SCHOOL

OLENDORF ED

OLENDORF AMY\&LEO

ANDERSON OSCAR

FOSS AIR AK\&FOSS JAM

FOSS JAMES\&FOSS AIR

BIG LAKE LODGE

SANDERS JAMES G

RUDY SARAH

PACK BILL

SUNSET TRLR PRK

DAVIS $W \quad K$

BURKESHORE MARINA M

PUHL RAY

PUHL RAYMOND

06-10-88

01-01-71

07-11-59
CORYELL MARSHALL WINCHESTER RAY BAKER
ASSIGNOR

OF OTHER

IDENTIFIER

UNION

UNION

ROCKY LK ALASKA

FARR SUB

LAS

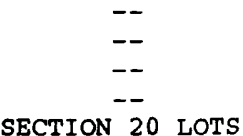

GOVT LOTS

--

FEULNER1968 OFR --

FEULNER1 968 OFR WSP1494

ROCKY LK SUB

LAS

FEULNER1968 OFR

HERMAN SUB

LAS

WSP1494

BÚTLER SUB ADO2

LAS

BUTLER SUB AD03

LAS

BUTLER SUB AD03

FISH CREEK ADD1 LAS

WSP1 494

HOBSON SUB

BIG LK AIRPORT

LAS

FEULNER1968 OFR GOTHBERG SUB

FEULNER $\overline{1968 \text { OFR }}$ FEULNER 1968 OFR BURKE SHORE SUB LAS

BURKE SHORE SUB BURKE SHORE SUB 
BIG LAKE - 3 MILE RADIUS

\section{LOCAL WELL NUMBER}

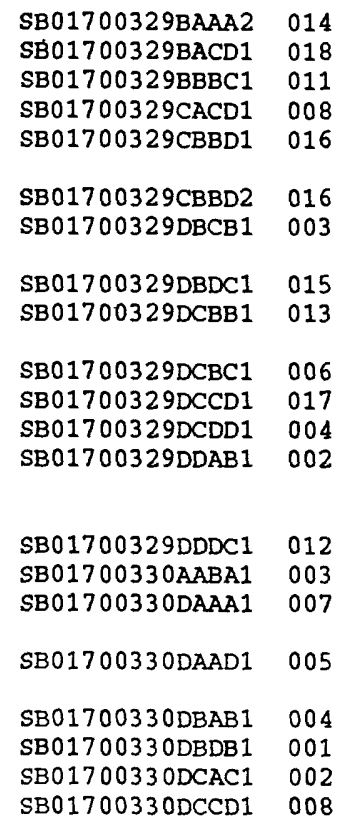

IDATE : $04 / 12 / 94$

LOCAL WELL NUMBER

$\begin{array}{ll}\text { SB01700330DDAD1 } & 006 \\ \text { SB01700331BADA1 } & 001 \\ \text { SB01700333ACBC1 } & 005 \\ & \\ \text { SB01700333ACCB1 } & 003 \\ & \\ \text { SB01700333BBBD1 } & 001 \\ \text { SB01700333BBCA1 } & 002 \\ \text { SB01700333BCBD1 } & 004 \\ \text { SB01700333CBAB1 } & 006 \\ & \\ & \\ \text { SB01700412DCAB1 } & 001 \\ \text { SB01700414ABAB1 } & 001 \\ \text { SB01700414ABAB2 } & 001 \\ \text { SB01700414ACAB1 } & 003 \\ \text { SB01700414ADCB1 } & 002 \\ \text { SB01700426ABDB1 } & 004 \\ \text { SB01700426ABDB2 } & 004 \\ \text { SB01700426ADAA1 } & 002 \\ \text { SB01700426CAAD1 } & 001 \\ & \\ \text { SB01700427DABC1 } & 001 \\ \text { SB01700435ACCD1 } & 001 \\ \text { SB01700435CDDD1 } & 002\end{array}$

\begin{tabular}{|c|c|c|c|c|c|}
\hline \multicolumn{6}{|l|}{$P R$} \\
\hline USE & DEPTH & WATER & DATE & & ASSIGNOR \\
\hline OF & OF WELL & LEVEL & WELL & & OF OTHER \\
\hline WATER & (FEET) & (FEET) & CONSTRUCTED & OWNER & IDENTIFIEI \\
\hline
\end{tabular}

\begin{tabular}{|c|c|c|c|c|c|}
\hline $\mathrm{H}$ & 101. & 31. & $06-08-88$ & SOULES GENE & BURKE SHORE SUB \\
\hline $\mathrm{H}$ & 101 & -- & $06-22-78$ & MOSBY WILBRT \& ANN & BIG LAKE SUB \\
\hline $\mathrm{H}$ & 50.4 & 20.00 & $02-22-71$ & OAKES NATHAN & -- \\
\hline $\mathrm{H}$ & 78.0 & 4.00 & -- & PETERSEN LESTER & -- \\
\hline $\mathrm{H}$ & 35.0 & 10.00 & $01-04-74$ & HELMS JACK & SPLINTER ADD \\
\hline $\mathrm{H}$ & 119 & 20.00 & $08-14-78$ & HELMS JACK & SPLINTER ADD \\
\hline $\mathrm{H}$ & 80. & -- & $-\quad-83$ & BROWN GREGORY\&JUANIT & A.A.FARMER \\
\hline & & & & -- & LAS \\
\hline $\mathrm{C}$ & 14.0 & -- & -- & PAYTONS POINT & WSP1494 \\
\hline $\mathrm{H}$ & 65.0 & 3.00 & $06-10-75$ & HOLT BOB & HOLT \\
\hline $\mathrm{H}$ & 67.0 & 4.00 & $06-11-75$ & PHILLIPS GLEN & -- \\
\hline $\mathrm{H}$ & 47.0 & -- & $09-03-77$ & LESTER JOSEPH L & -- \\
\hline $\mathrm{P}$ & 100 & -- & $01-01-70$ & AK DIV PKS BIG LAKE & -- \\
\hline $\mathrm{H}$ & 50. & 10 & $06-08-79$ & BRINGMANN GEORGE & HIBBARD ADD \\
\hline
\end{tabular}
-- LAS

$06-28-76$

$01-14-75$

02-12-79

01-02-74

12-14-73

$06-29-76$

$06-30-76$

$06-18-86$

$38.4 \quad 15.00$

$180 . \quad 150$.

BIG LAKE - 3 MILE RADIUS

SMITH MARVIN

FARR DAN

EASTBERG ED\&CAROL J LEWIS SUB

LAAK WILLIAM

LAS

SHUPE MIKE

WINCHESTER RAY

LANZ JIM

KREWETZKI HORST

DATE

PRIMARY

$\begin{array}{llll}\text { USE } & \text { DEPTH } & \text { WATER } & \text { DATE } \\ \text { OF } & \text { OF WELL } & \text { LEVEL } & \text { WELL }\end{array}$

WATER (FEET) (FEET) CONSTRUCTED

$05-22-76$
$01-01-66$

$01-01-66$
$10-17-70$

$\begin{array}{ccll}\mathrm{H} & 183 & 80.00 & 05-22-76 \\ \mathrm{C} & 42.0 & -- & 01-01-66 \\ \mathrm{H} & 93 . & 22 . & 10-17-70 \\ \mathrm{H} & 32.0 & 20.25 & 06-22-72 \\ & & & \\ \mathrm{H} & 115 & -- & 01-01-70 \\ \mathrm{H} & 80.0 & 40.00 & 06-01-76 \\ \mathrm{H} & 63 . & 36 . & 10-03-81 \\ \mathrm{H} & 94 . & 30 . & 06-10-86\end{array}$

BIG LAKE YCHT CLB

KLOUDA JOE\&PAT

RAPP DICK

KRULL MARION

WILDE LAWRENCE

HANSEN ROGER

NECRASON CONRAD\&MYRL

$01-01-54$

$06-28-76$

$07-23-78$

$06-10-86$

$03-26-83$

$09-19-78$

06-03-80

$05-25-76$

$05-01-73$

CAMPBELL HOWARD

KORMAN DARRELL

PETERSON HANK

JOHNS MARIE\&ROBERT

GIST PERCY

GRANUS WALTER

GATES HERB.\& KATH.

$03-\overline{24}-71$

$1 \overline{6.00}$

$07-01-75$
OWNER

COOK WALTER

$\mathrm{K}^{--}$

$$
\text { -- LAS }
$$

FRIDLEY DAVE

UNREIN JOHN

CALL OF WILD CP

HOYT HARRY

ASSIGNOR
OF OTHER
IDENTIFIER

ST́ARBOARD COVE FEULNER1968 OFR ECHO LAKE PARK LAS

ECHO LAKE PARK

ECHO HILLS SUB ECHO HILLS SUB SECTION 33 LOTS

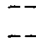

HORSESHOE LAKE BYRNE SUB DAVIS SUB DAVIS SUB DAVIS SUB MCINTIRE BETTY

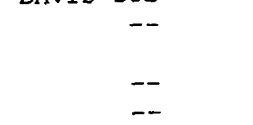

KUKOWSKI SUB 
BIG LAKE VILLAGE - 2 MILE RADIUS

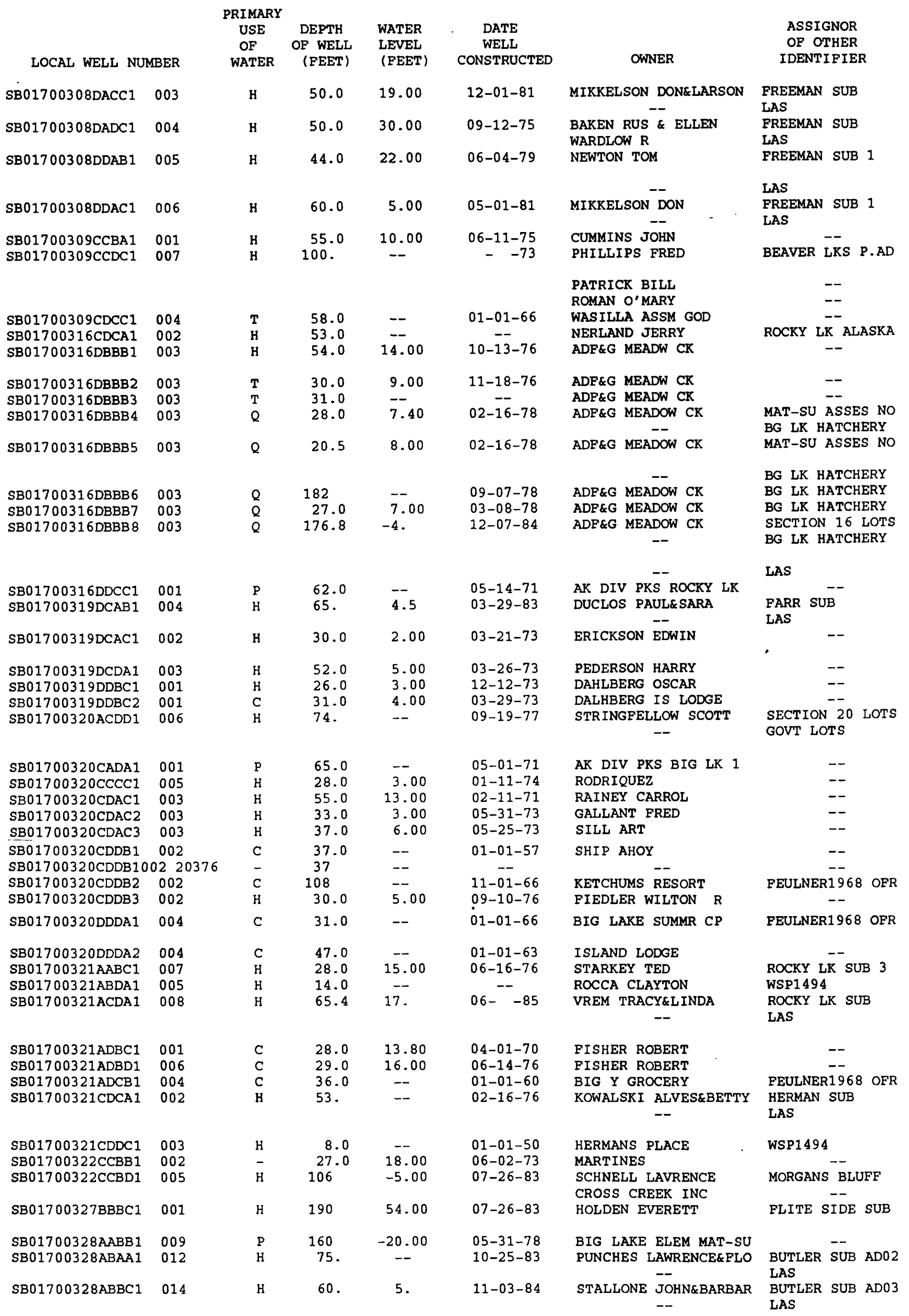


LOCAL WELL NUMBER

SB01700329ABAD1 005

SB01700329ABBA1 019

SB01700329ABBB1 010

SB01700329ABDB1 007

SB01700329BAAA1 014

SB01700329BAAA2 014

SB01700329BACD1 018

SB01700329BBBC1 011

SB01700329CACD1 008

SB01700329CBBD1 016

SB01700329CBBD2 016

SB01700329DBCB1 003

SB01700328ABDB1 008

SB01700328ACAA1 007

SB01700328ACBC1 002

SB01700328ACCB1 006

SB01700328ADAC1 013

SB01700328BABA1 004

SB01700328BACD1 010

SB01700328BCDC1 001

SB01700328BDBB1 005

SB01700328DBBB1 003

SB01700329AAAB1 001

SB01700329AABA1 009

SB01700329DBDC1 015

SB01700329DCBB1 013

SB01700329DCBC1 006

SB01700329DCCD1 017

SB01700329DCDD1 004

SB01700329DDAB1 002

SB01700329DDDC1 012

SB01700330AABA1 003

SB01700330DAAA1 007

SB01700330DAAD1 005

SB01700330DBAB1 004

SB01700330DBDB1 001

SB01700330DCAC1 002

SB0170033 ODCCD1 008

SB01700330DDAD1 006

SB01700331BADA1 001

SB01700333BBBD1 001

SB01700333BBCA1 002
USE

WATER

DEPTH

WATER

DATE

WELL

CONSTRUCTED

ASSIGNOR

OF OTHER

(FEET)

$\begin{array}{lllll}H & 30.0 & 8.00 & 01-01-71 & \text { PUHL RAY } \\ H & 89.6 & - & 06-03-77 & \text { PUHL RAYM }\end{array}$

89.6

$--$

$06-10-88$

01-01-71

07-11-59 BAKER

$\begin{array}{rrr}67.0 & 15.00 & 07-11-59 \\ 101 & 31 & 06-08-88\end{array}$

$101 . \quad 31$.

101

$-$

$06-22-78$

$50.4 \quad 20.00$

$02-22-71$

78.0

4.00

$--$

$35.0 \quad 10.00$

119.20 .00

$01-04-74$

$08-14-78$

$-\quad-83$

$74.0 \quad 15.00$

$-$

16.

06-30-75

$--$

50. 16 .

05-24-85

16.0

$-$

75.

20.

11-01-85

01-01-61 $10-25-79$

\section{$\mathrm{P}$}

$82.3-2.00$

$$
--
$$

14.

$99.5 \quad 22.00$

$05-05-75$

$07-01-67$

$\begin{array}{lll}14.0 & -- & 07-14-67\end{array}$

$60.0 \quad 41.00 \quad 01-01-64$

14.0

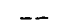

65.0

$-$

67.0

3.00

47.0

4.00

$06-10-75$

06-11-75

09-03-77

100

50.10 .

$06-08-79$

98.6

18.00

$06-28-76$

28.0

6.00

01-14-75

52 .

5.

02-12-79

$$
\begin{array}{rr}
45.0 & 18.00 \\
75.0 & 40.00 \\
42.0 & 13.00 \\
38.4 & 15.00 \\
180 . & 150 .
\end{array}
$$

01-02-74

12-14-73

06-29-76

$06-30-76$

06-18-86

$\begin{array}{cc}183 & 80.00 \\ 42.0 & - \\ 115 & - \\ 80.0 & 40.00\end{array}$

$05-22-76$

$01-01-66$

01-01-70

06-01-76
OWNER

CORYELL MARSHALL

WINCHESTER RAY

SOULES GENE

MOSBY WILBRT \& ANN

OAKES NATHAN

PETERSEN LESTER

HELMS JACK

HELMS JACK

BROWN GREGORY\&JUANIT

WINCHESTER MIKE

BIG LAKE SCHOOL

OLENDORF ED

OLENDORF AMY\&LEO

ANDERSON OSCAR

FOSS AIR AK\&FOSS JAM FOSS JAMES\&FOSS AIR --

IDENTIFIER

BURKE SHORE SUB

LAS

BURKE SHORE SUB

$-$

BURKE SHORE SUB BURKE SHORE SUB BIG LAKE SUB

$$
-
$$

SPLINTER ADD SPLINTER ADD

A.A.FARMER

BUTLER SUB AD03

FISH CREEK ADD1 LAS

WSP1494

BIG LK AIRPORT

LAS

FEULNER1968 OFR

SANDERS JAMES G

GOTHBERG SUB

RUDY SARAH

PACK BILL

SUNSET TRLR PRK

DAVIS $W K$

BURKESHORE MARINA M FEULNER1968 OFR

PAYTONS POINT

WSP1494

HOLT BOB

PHILLIPS GLEN

LESTER JOSEPH I

AK DIV PKS BIG LAKE BRINGMANN GEORGE

HOLT

$$
--
$$

FEULNER1968 OFR

SMITH MARVIN

FARR DAN

EASTBERG ED\&CAROL J

SHUPE MIKE

WINCHESTER RAY

LANZ JIM

KREWETZKI HORST

COOK WALTER

BIG LAKE YCHT CLB

KRULL MARION

WILDE LAWRENCE

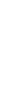

HIBBARD ADD EXT

LAS

LEWIS SUB
LAS

STARBOARD COVE STARBOARD COVE STARBOARD COVE STARBOARD COVE STARBOARD COVE

LAS

STARBOARD COVE FEULNER1968 OFR --

ECHO HILLS SUB
LAAK WILLIAM 
Walter Weaver

URILLLILS LUG

Driller G \& G DRILLING $\infty$. - LeeH. Gohr

sethermath

;

Hax. Wablets

ivell so.

Iddress_ Walter We Anchorage, Alaska $_{2}$

Date started July 16, 1956

Date completed July 26, 1956

Use of well dowe

Location of well 8 Miles from Wasilla on Big Lake Road

Depth of well_z3 feer.

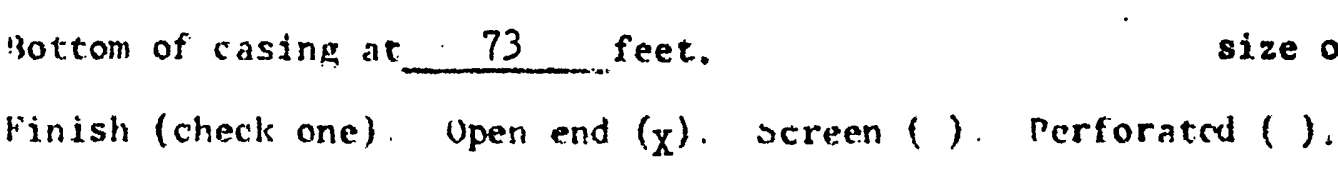

Describe screen or perforations

Static wath level $29 \quad$ feet (above) (below) land surface.

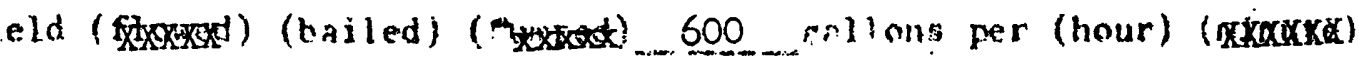

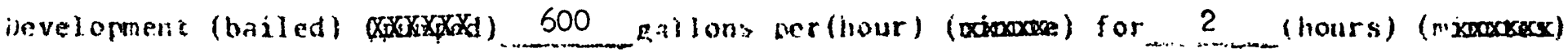

with 10 fert of itrantewn

hescription of formation (descrabist material, inlor, whether hard or soft, water bearing, etr.

Top Sojl

Clay \& Gravel Hard

Blue Clay Soft

Gravel $\hat{\alpha}$ 'ome Water

Blue Clay Soft

Water Bearing_Gravel

\begin{tabular}{|c|c|c|c|}
\hline Thickness & \multicolumn{2}{|c|}{ ilepth } \\
\cline { 2 - 3 } of formation & Trom & To \\
\hline 3 & 0 & 3 \\
\hline 32 & 3 & 35 \\
\hline 23 & 35 & 58 \\
\hline 2 & 58 & 60 \\
\hline 11 & 60 & 71 \\
\hline 2 & 71 & 73 \\
\hline & & \\
\hline
\end{tabular}




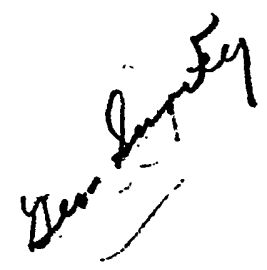

LFILILERS LOQ

$\because$ : Ber

Q \& a DRILLTNa CO Elee $\mathrm{H}$. Gohr

vell. Owner JIM POTTER

BOX 153, Anchorage, Llaska

Hddress

f. Poth

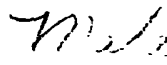

Date started

$8 / 29 / 58$

Date completed

$8 / 21 / 58$ Use of well

Location of well/Township, Range \& Section, if known or distanks. from main roads)

2 Miles Right of Pittman

Total depth of drilled well 38 peet

Bottom of casing at 38 feet Size of casing

Finish (Cheok one) Open ond $F^{\pi}$ ) Screen ( ) Perforated ( )

Describe screen or perforations

Well development (balled) or ( (minute) for 3 (hours)(m15utes) with 33 feet of drawiow: Static water level. Ieet (abovel (below) land surface Remarks

\begin{tabular}{|c|c|c|}
\hline $\begin{array}{l}\text { Description of formation thpe of } \\
\text { material, hard or sort, weter bearing, }\end{array}$ & Thickness & $\frac{\text { Depth }}{\text { From to }}$ \\
\hline Top silil \& Sand (Brown) & $\mathbf{I H}_{\mathrm{i}}$ & 0-I4 \\
\hline Sand \& Pea Gravel (Water Bearing) & 5 & Th-19 \\
\hline \multirow{6}{*}{ Clay with seams of water bearing gravel } & 19. & $19-38$ \\
\hline & & \\
\hline & $\cdot$ & \\
\hline & & \\
\hline & & \\
\hline & & 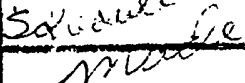 \\
\hline
\end{tabular}


T212er G \& G DRILIING CO. - Lee H. Gohr

Ownor K. Lovejoy

Adress St. Rt. Wassila

Iooation of weil Approx 5 miles past Pittman
Date Started Aug 11, 1958

Däto comploted Aug. 14, 1958

Dse of well Dairy

Depth of well 35 seot.

Bottom of casing at 35 foot. .

o180 of casing. 6" Hvy

Finish (oheok ono). Open end $(x)$, Sereen ( ). Perforated ( ).

Describe screen or perforations

Static water lovel $20 \frac{1}{2}$ foet (abow) (below) Iand surface.

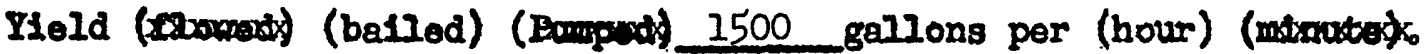

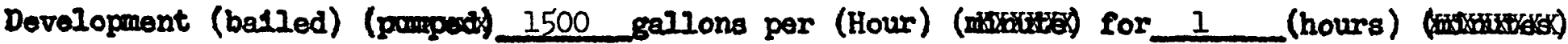
with 3 feot of drasdown.

Desoription of formation (desoribing material, color, whether hard or soft, water bearing, etc.

Top Soil

Sand $\&$ Cravel

Water bearing Sand \& Gravel

(Didnot go to bottom of sand \& gravel)

L. Gohr

\begin{tabular}{|c|c|c}
\hline \multirow{2}{*}{ Th1oknoss } & \multicolumn{2}{|c}{ Depth } \\
\cline { 2 - 3 } & From & To \\
\hline 26 & 0 & 1 \\
\hline 8 & 1 & 27 \\
\hline & 27 & 35 \\
\hline & & \\
\hline & & \\
\hline & & \\
\hline
\end{tabular}




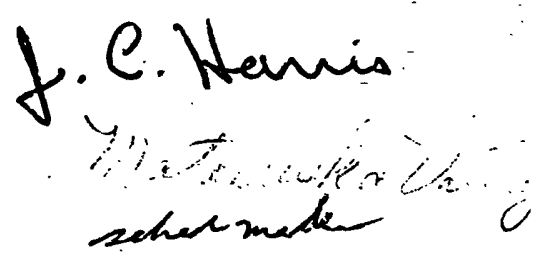

\section{IFIILLERS IOOG}

Ler G \& G DRILLING CO. Lee H. Gohr Date started 8-15-58.

rell Omer J. C. Harris

Date completed $8-17-58$

r.didress One Mile from Pittman Big Lake Rd Use of well Home

Location of well(Townsh1p, Renge \& Section, if known or distanc. from main roads)

Total depth of drilled well 43 peet

Bottom of casing at 43 feet Size of casing 6"

Finlsh (Cheok one) open end $(x)$ Screen ( ). Perforated ( )

Describe soreen or perforations

Well development (ballea) or ( 1200 gelions per(houx)

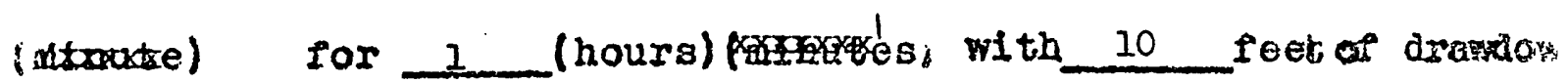
Static water level 16 feet (a (below) land surface Remarks_Sand \& Pea gravel heaves in pipe 28 to $40 \mathrm{ft}$

\begin{tabular}{|c|c|c|}
\hline $\begin{array}{l}\text { Description of formation (type of } \\
\text { material, hard or soft, water bearing. } \\
\text { color etc.) }\end{array}$ & Thl ckness & $\frac{\text { Depth }}{\text { From to }}$ \\
\hline Top soil & 7 & 021 \\
\hline Sand \& Gravel & 20 & $1-21$ \\
\hline Water bearing sand \& gravel & 7 & $21-28$ \\
\hline Water bearing sand ( $F$ 'ine) & 10 & $28-38$ \\
\hline Pea Gravel & 2 & $36-40$ \\
\hline In rge $3 \times x d x d x$ ary Gravel $\&$ Sand & .3 & $40-43$ \\
\hline Didnot go to bottom of stream & & \\
\hline & & \\
\hline & & \\
\hline
\end{tabular}


M-W DRILLING, InC.

Houston sheet 18

P. O. Box 4-1728 - 2811 Dawson

A C 907-279-1741

ANCHORAGE, ALASKA 99509

\section{DRILLING LOG}

Well Owner.

Greet Plains (American) Inc.

Use of Well com.

Location (address of: Township, Range, Section, if known; or distance main road Horstioe Lake, Gr Plains XXXX Noel state 1 ; well site Eig La'se Area

Size of casing_ 6 Depth of Hole 93 feet Cased to 93 feet

Static water level 7 ft. (尚ơve) (below) land surface. Finish of well (check one) open end ( $X$ ); Screen ( ); Perforated ( ).

None

Describe screen or perforation

Well pumping test at 60 gallons per (hoư⿱ of drawdown from static level.
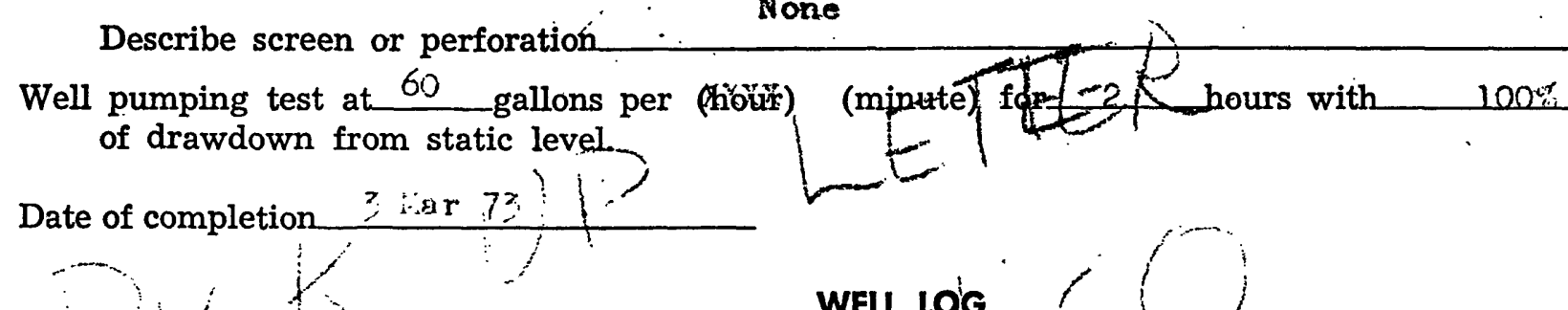
$100 \%$ ft.

\section{Depth in feet-from} ground surface
Give details of formations penetrated, size of material, color and hardness

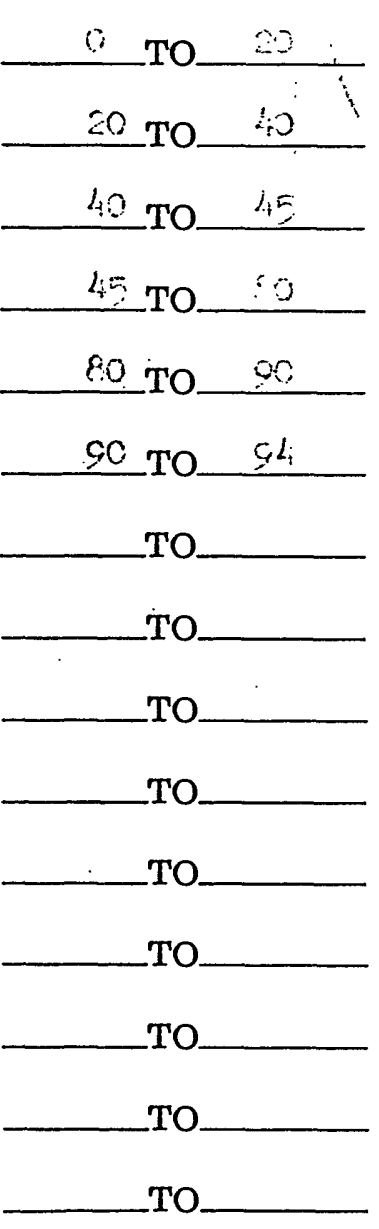

Tine Grey Sand

Sand: Grey, wet--etatic level 151

Sand $a / a$

Sedium to cosrse Gravel: slifhtly sandy, good water--20 U. T.

Corrse Grovel: excellent weter 60 Crit.

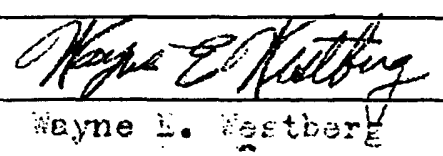




$$
\begin{aligned}
& \text { Ed.L. Q ngram } \\
& \text { area } \\
& \text { sehed Made Vallow }
\end{aligned}
$$

Owner Ed. I. Ingram

Use of well Home \&usiness

Location LAddress or; Wunship, Kange \& Section if thown; or alstance from iain roads) Big Lake $Y$.

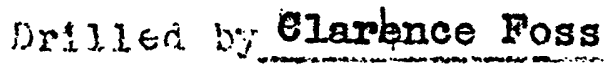

Dete con 1 leted $7-74-59$

bottoni or ciasting it 32

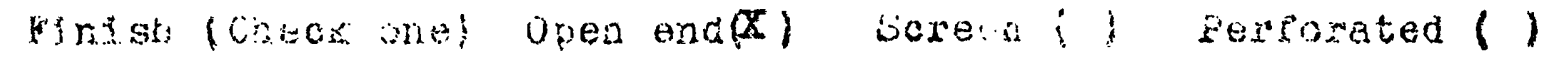
4icixes Swafford Drilling Co. Total aiph of drilled hole 33 ft: feot bize of casins 6"

3401 Spenard Road

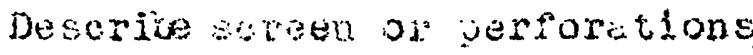

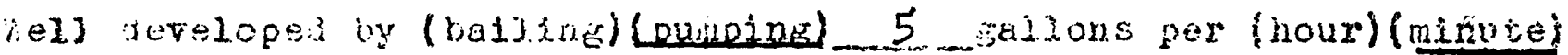
for 2 (bursifoinutes) with 10 feet of drawdom

sutic water lersl

\begin{tabular}{|c|c|c|}
\hline 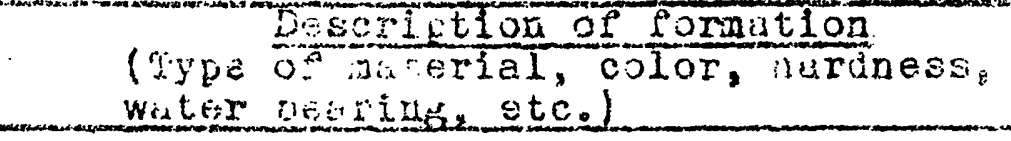 & Toicrnesi & Deptin \\
\hline Sand and Clay (Brown) & 15 & $0-15$ \\
\hline Clay (Blue) and Gravel & 15 & $15-30$ \\
\hline Sand $w / H^{2} O$ at 30 feet. & 3 & $30-33$ \\
\hline & & \\
\hline & & \\
\hline & & \\
\hline & & \\
\hline & & \\
\hline . & & \\
\hline
\end{tabular}
20 feet inovelledow land surface Reners Pumped full capacity of rell from $30 \mathrm{ft}$ 
:AATER YELL DR:LLI:RS LOG

Drising co. Moffitt

Driller

Well owner Apelph Mutine;
DO NOT FILL IN

USGS No.

Area

Use of ivell

Location (address of: Township, Range, \& Section (if known); distance from road: Big Lake Bo He 20 Anch 99503

size of Casing 6 Depth of Hole 30 feet. Cased to 30 feet.

Static water ievel $/ 8$ feet (above) (below) land surface. Finish of well

(check one) Open end ( $)$ : Screen ( ): Perforated ().

Describe screen or perforations:

Hell pumping test at $5-7$ gallons per (hr) (min) for feet of drawdown from static level. hours with

Remarks

$6-5-73$

HELL LOG

Depth in feet from Give details of formations penetrated, size of material, ground surface color, and hardness.

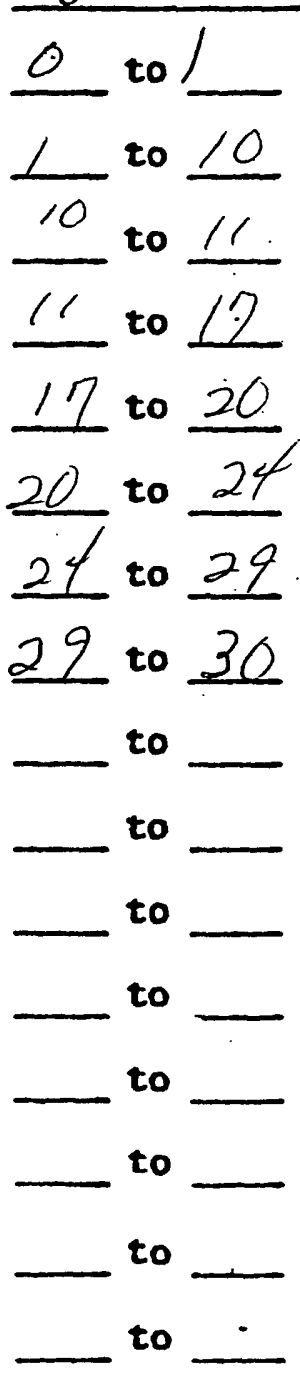
ine 


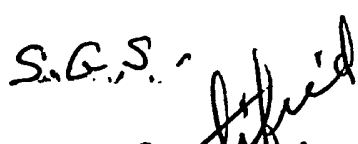

colity

Hontom me sielc

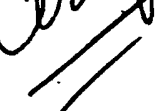

i

DRSIIESS TOG

(For tasces upe only

sechol madel

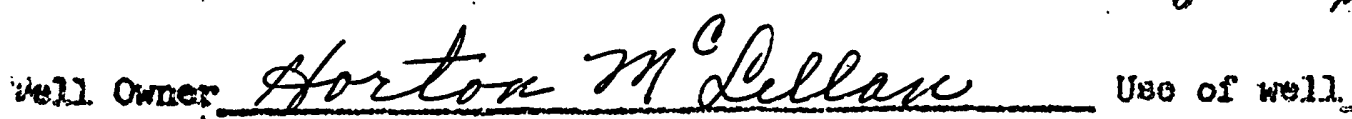

Iocation (Address of; Tomship, Range \& Section, if lanown; or distance from main road

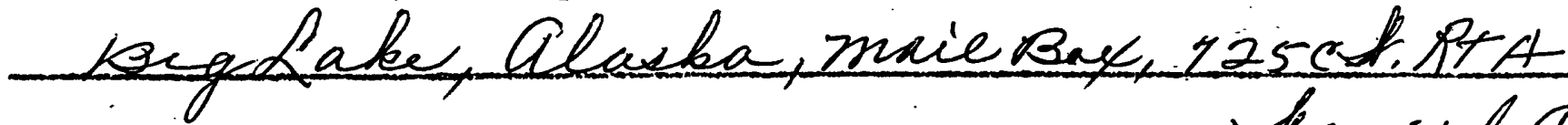

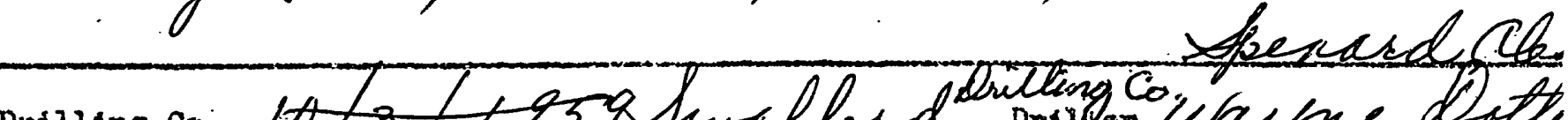

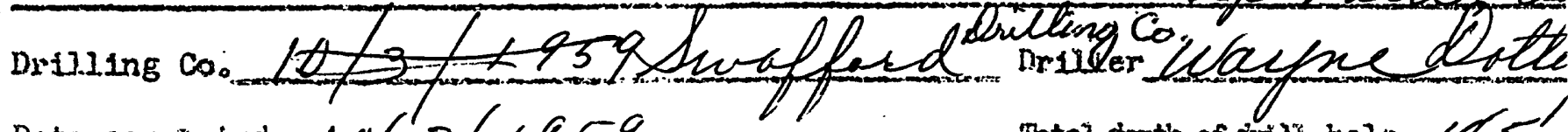
Dete completed $\angle 0 / 3 / 1959$ Bottion of casing at. Total depth of oxill hole $\frac{145}{1 / 2}$ Finiah (Chock one) open enci $X$ : Screen ( ); Perforated ( )

Describe gareun or perfonitane.

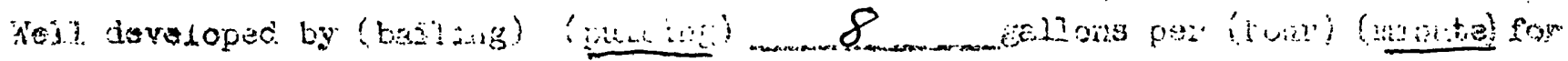

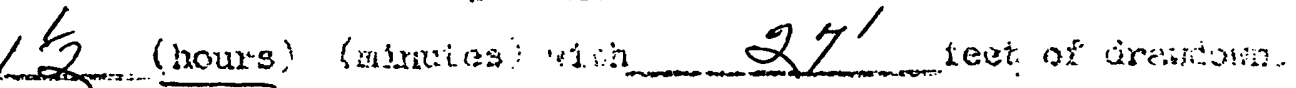

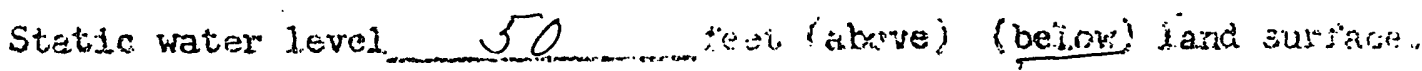
Risinarks

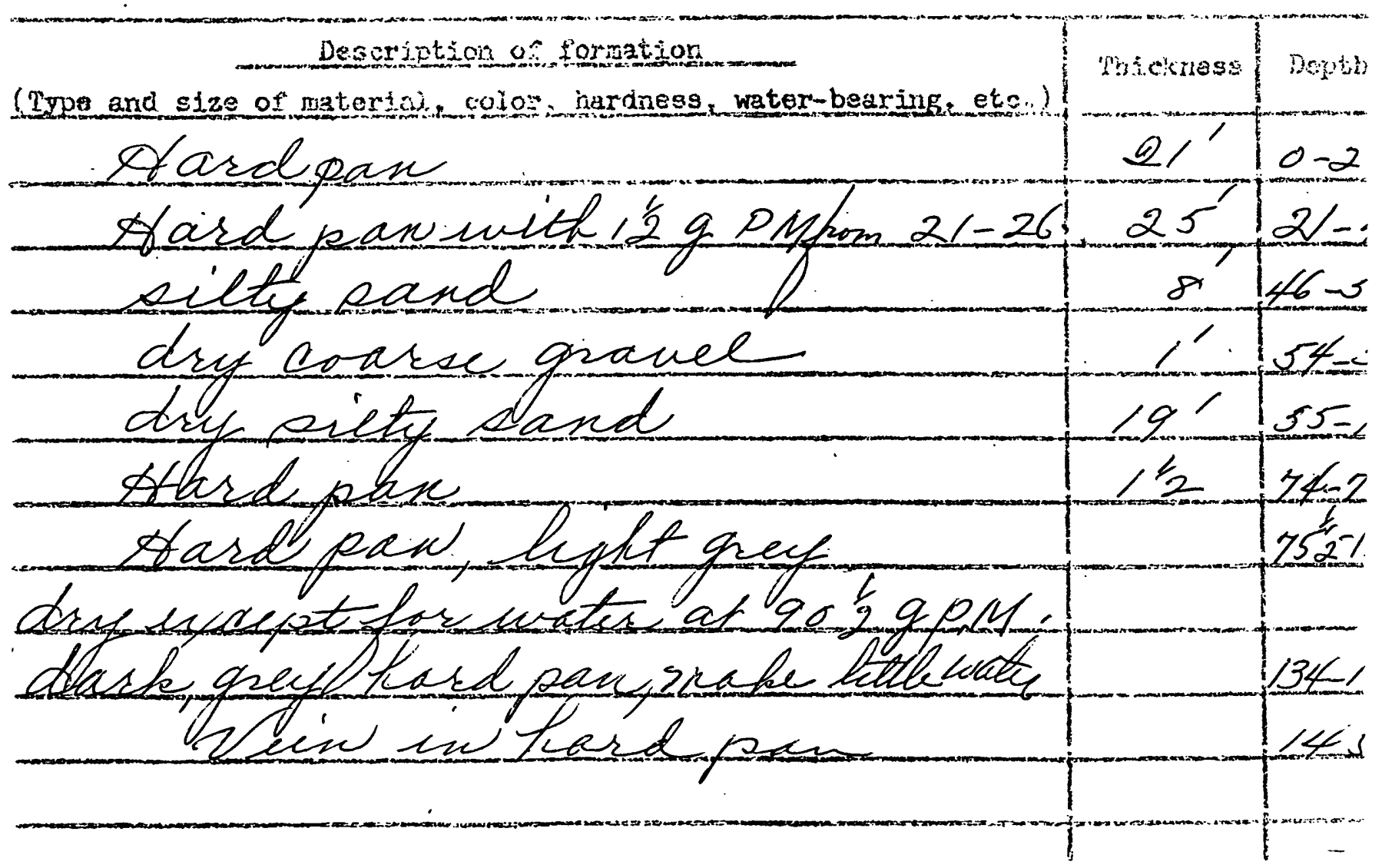


Driling co. Mofyitt

Driller USGS No. DO NOT FILL IN

Hell owner Tic secengle

Location (address of: Township, Range, \& Section (if known); distance from road: Kig Lake Plea

size of Casing 6 Depth of Hole $4 / f$ feet. Cased to $4 / 4$ feet.

Static water level

foet (above) (below) land surface. Finish of well

(check one) Open end (r): Screen ('): Perforated ().

Describe screen or perforations:

ivell pumping test at 10 gallons per (min) for hours with feet of drawdown from static level.

Remarks

$2-\mid 9-21$

IVELL LOG

Depth in feet from ground surface Give details of formations penetrated, size of material, to $/ 2$ color and hardnessa

$\frac{0}{12}$ to $\frac{12}{17}$
$\frac{17}{22}$ to $\frac{22}{24}$
$\frac{24}{42}$ to $\frac{42}{43}$
43 to $\frac{44}{42}$

graml

haspaie

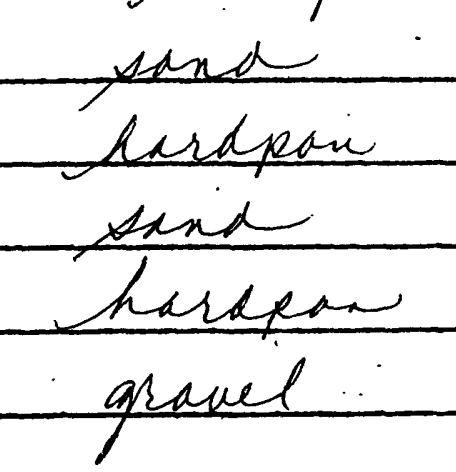

to

to

to

to

to

to

to

to

to 


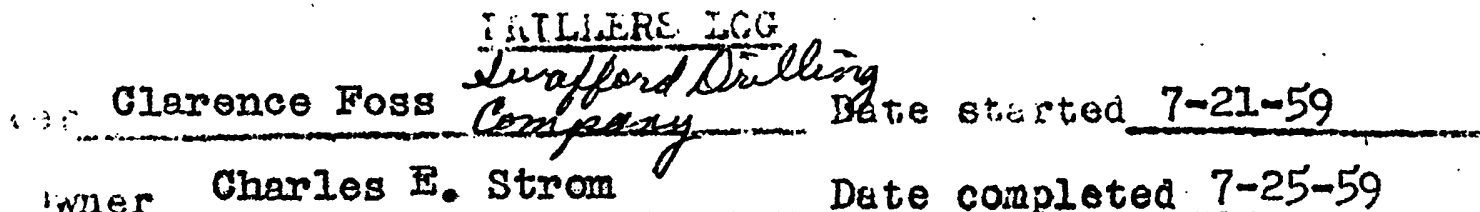
- : iwer Charles E. Strom Dete completed $7-25-59$ Adress 604 Hanor Ave,--Gov'mt. Hill Use of well Home Socation of well(Townshlp, Range sc Section, if known or distan jrom main roads) section 33-TITN--R3W--- scho Laken Big Lake CAPrea.-

Totil depth of drilled well 87 feet

Eotitom of casing at $87^{\text {feet }}$ size of casing $6^{n}$.

Finish (Cheok one) Open ond ( $)$ Soreen () Perforated ( ) liescribe screen or perforutlons

well development (balled) or (plimped) 9 gallons per (hour)

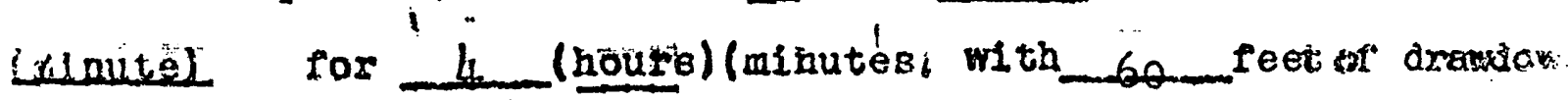
Static water level I5 feet (abovel (below) land surfece

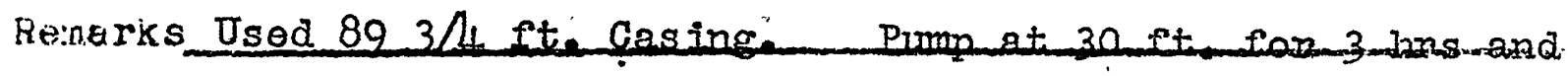
vater would not clear upe.

\begin{tabular}{|c|c|c|}
\hline $\begin{array}{l}\text { Doscription of rormation thpo of } \\
\text { meterial, hard or soft, water bearing. } \\
\text { color oto.l }\end{array}$ & Thlokness & $\frac{\text { Depth }}{\text { From to }}$ \\
\hline Clay (Brown) and Rocks & 74 & $-\Omega_{-14}$ \\
\hline Olay (BIue) w/Sand and Gravei & $j_{4}$ & $-14=28$ \\
\hline Clay and GraveI (Birty) w/H20-12 Gip.Mi & 3. & $28-31$ \\
\hline Glay (Blue) $w /$ Sand and Gravel & $\overline{6}$ & $37-37$ \\
\hline Clay (Blue) & 4 & $37-41$ \\
\hline Clay $\mathrm{r} / \mathrm{Sand}$ and Gravel & 27 & $47-68$ \\
\hline Hard-pan & 16 & $68-84$ \\
\hline Gravel $\pi / H^{2} O$ & 3 & $84=87$ \\
\hline & & \\
\hline & & \\
\hline
\end{tabular}




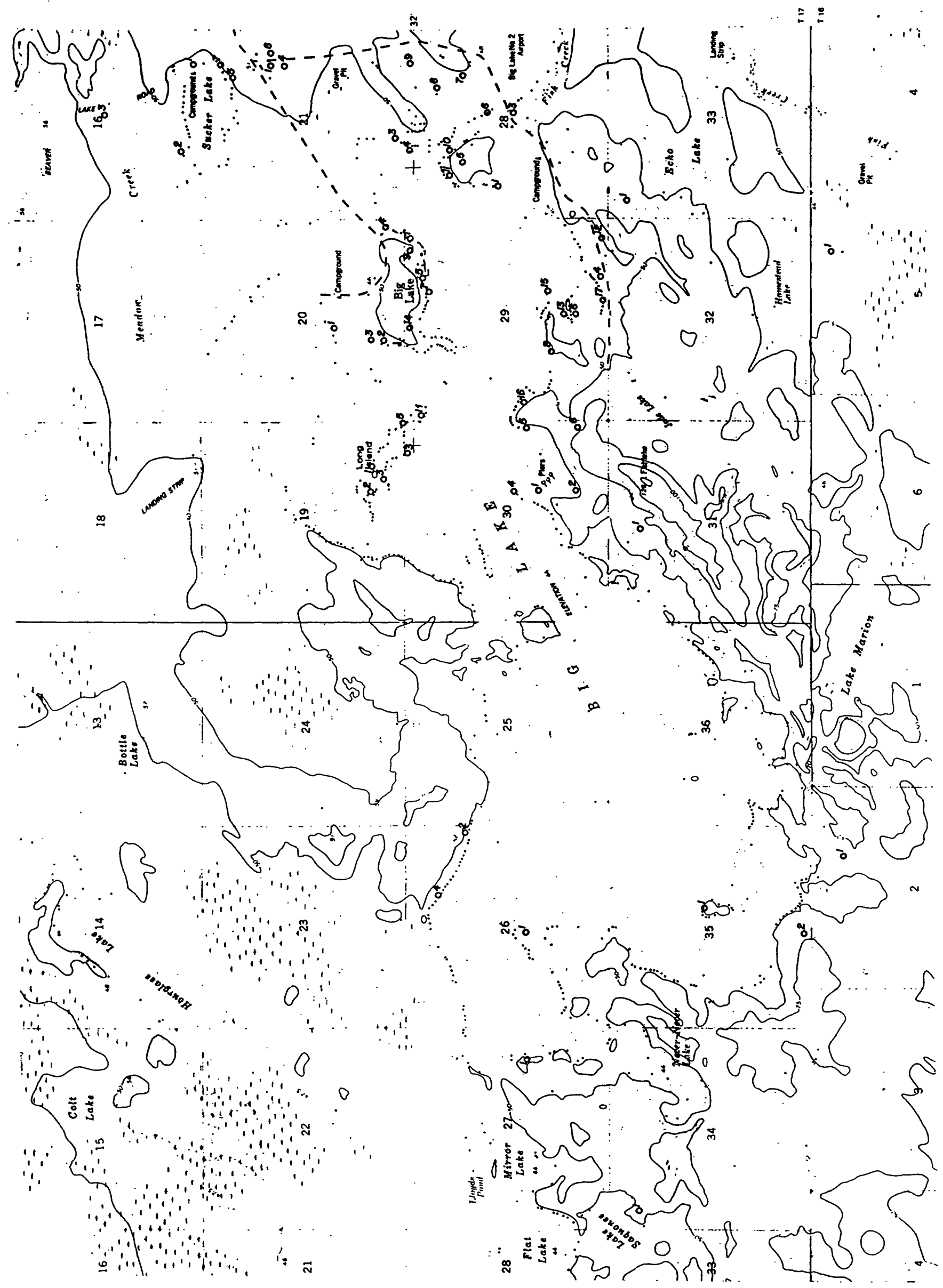

This figure corresponds with the following table(s). 




This figure corresponds with the following table(s). 


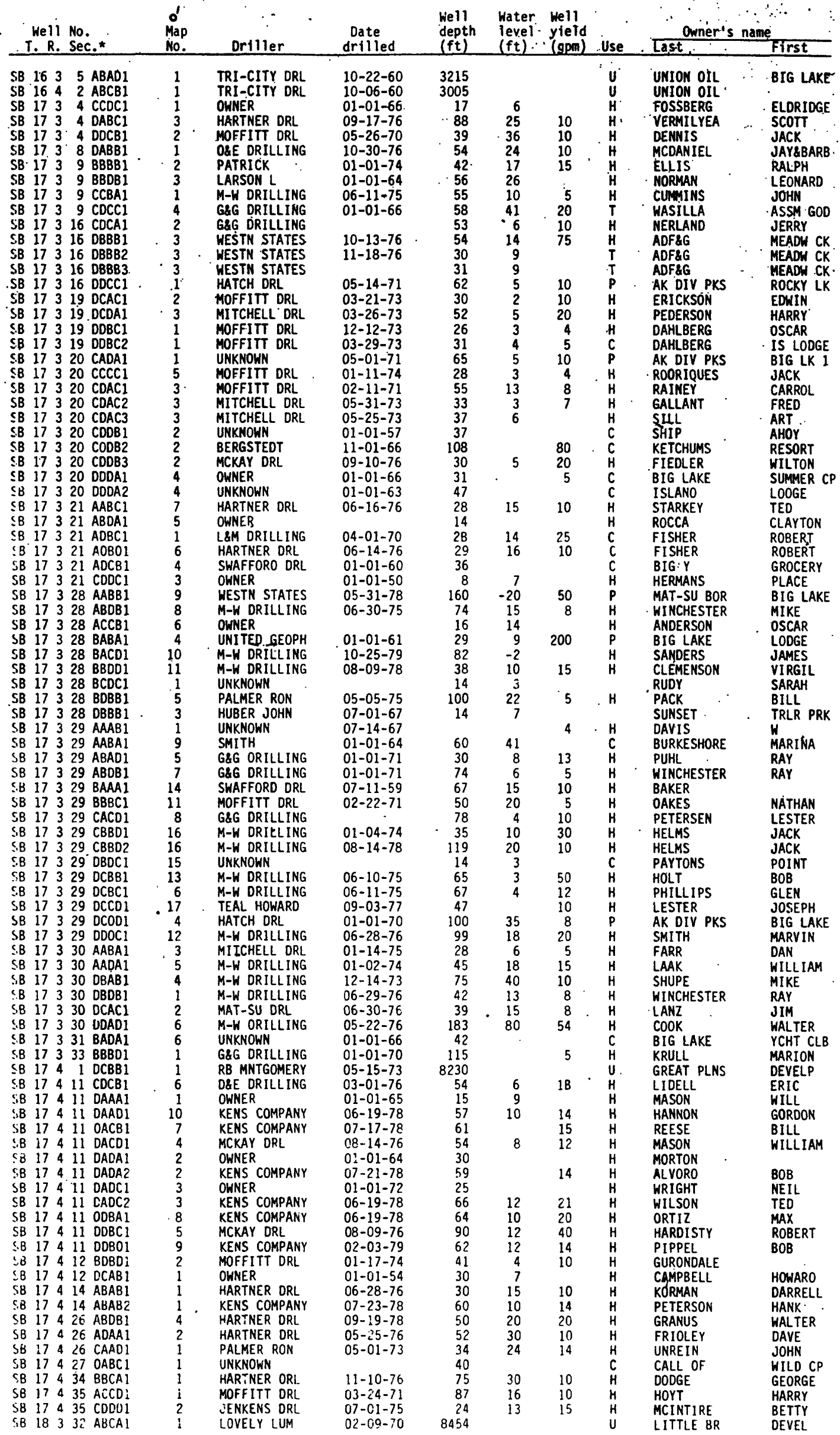




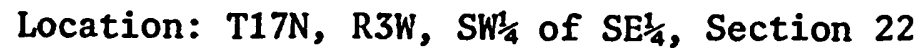

USGS map Anchorage (C-8) SE, Scale 1:25,000

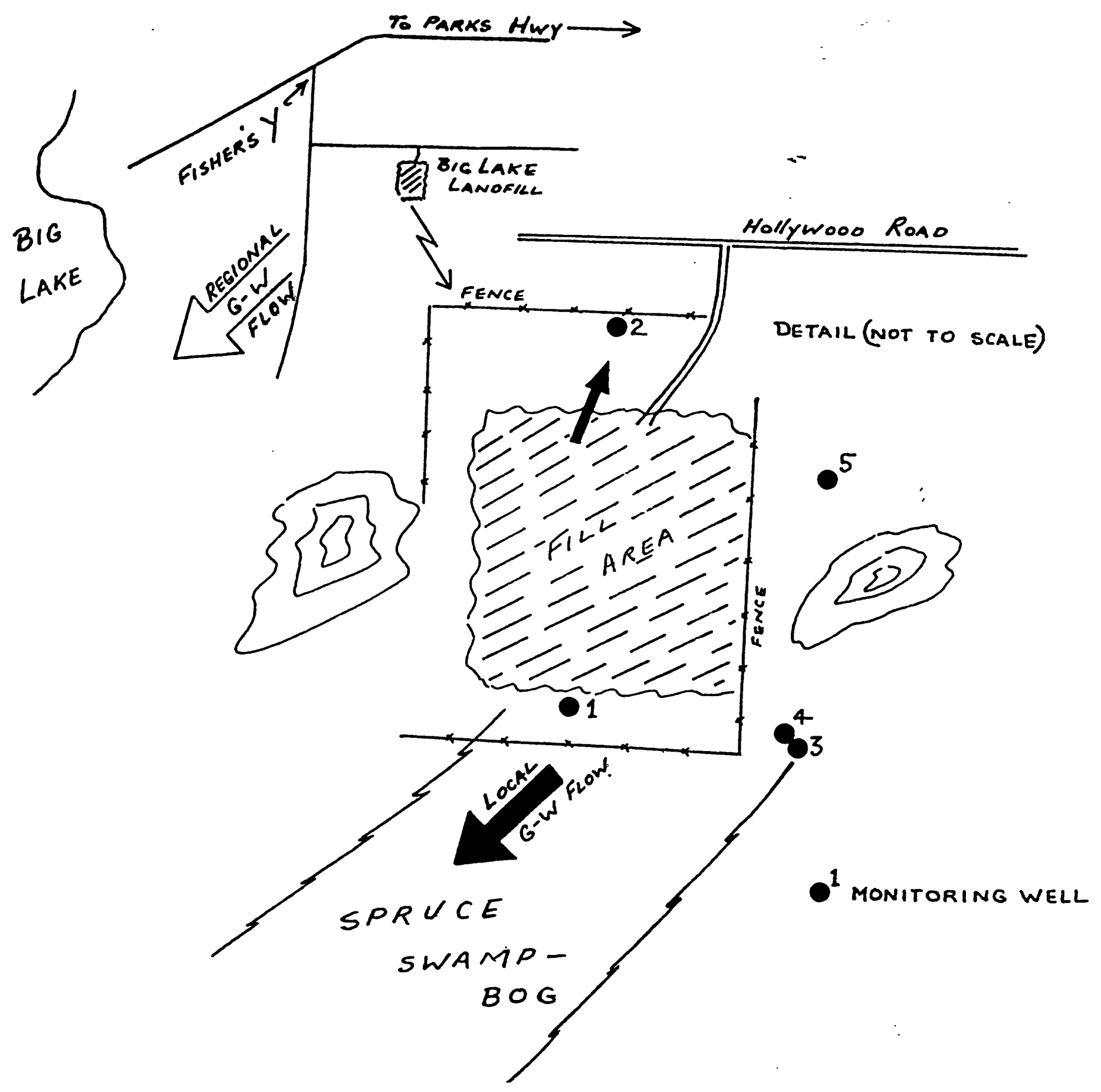

This figure corresponds with the following information. 
BIG LAKE SITE -- Mat-SU Landfills

Location: SW $1 / 4$ of SE $1 / 4$, Sec 22 , T17N, FiSW

Anchorage (C-8) SE sheet, scale 1:25,000 Approx $3 / 4 \mathrm{mile}$ east of paved road around east shore of Big Lake and $3 / 4 \mathrm{mile} N E$ of Big Lake airport No. 2

General topographic setting: Folling or hummocky terrain, morainal ridges surrounded by and cut by stream outwash deposits.

Ground-water flow direction: Regional flow direction to southwest and south, toward Big Lake, Fish Creek and ultimately to knik Arm. Apparent local flow at landfill is southwest through low, boggy spruce-covered area. Slope of active landfilling area in mid-to late-1985 possibly leads to surface and shallow groundwater flow to the northeast.

Onsite or nearby ground-water information: Domestic wells within a mile or two from site range in depth from a few tens of feet to about 100 feet; water levels are commonly less than 20 feet below land surface, and several deeper (about 100 feet) wells near Big Lake reportedly flow at the surface.

Information on ground-water conditions within the site is available from a 1982 report by Arctic Engineering Inc. Five 25-foot borings were made; none encountered ground water. Two wells were drilled:

No. 1 - Depth 85 feet, confined water-bearing zone at $58-59$ feet, $W L=24$ feet

USGS designation: Big Lake LF-S

USGS ID $613239 \quad 149471901$

No. 2 -- Depth 70 feet, water-bearing zones at 59-60 and $69-70$ feet, $W L=12$ feet

Monitoring wells: Four water-quality monitoring wells were drilled in February 1986. Drill logs and construction data follow. 


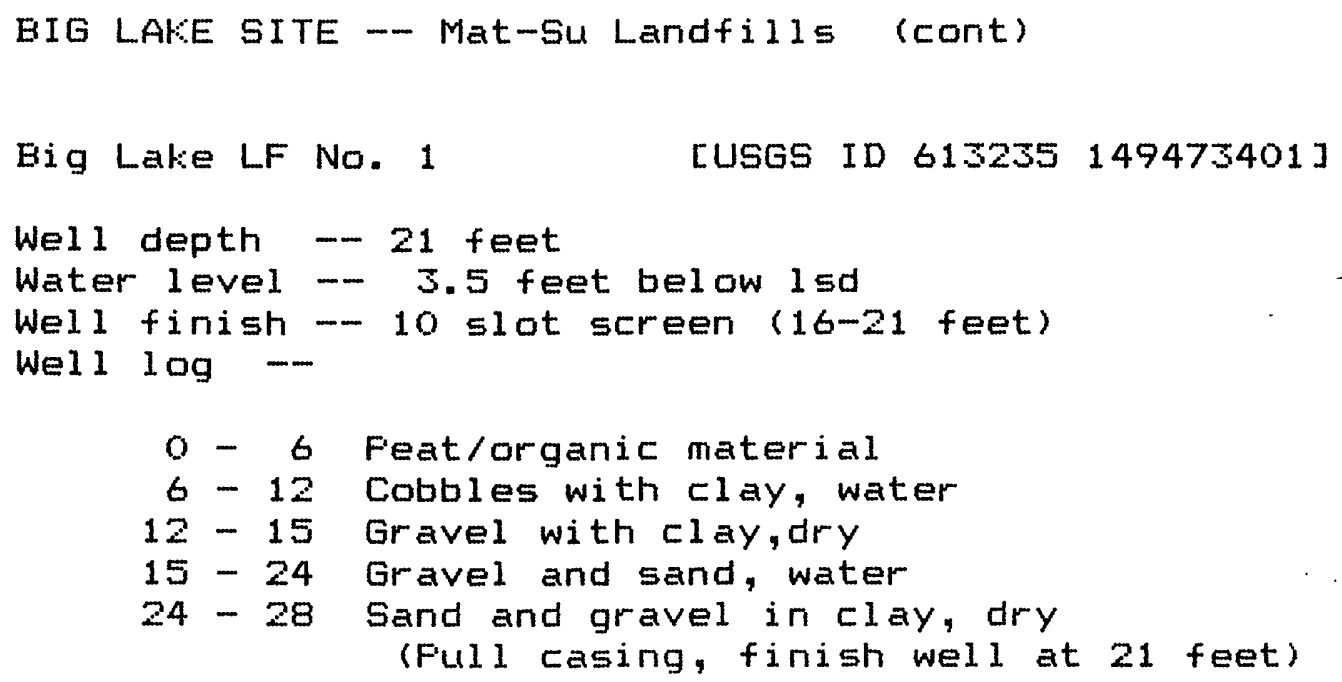

Eig Lake LF No. 2

Well depth - 65 feet

Water level -- 30 feet below lsd

Well finish - 12 slot screen (60-65 feet)

Well log --

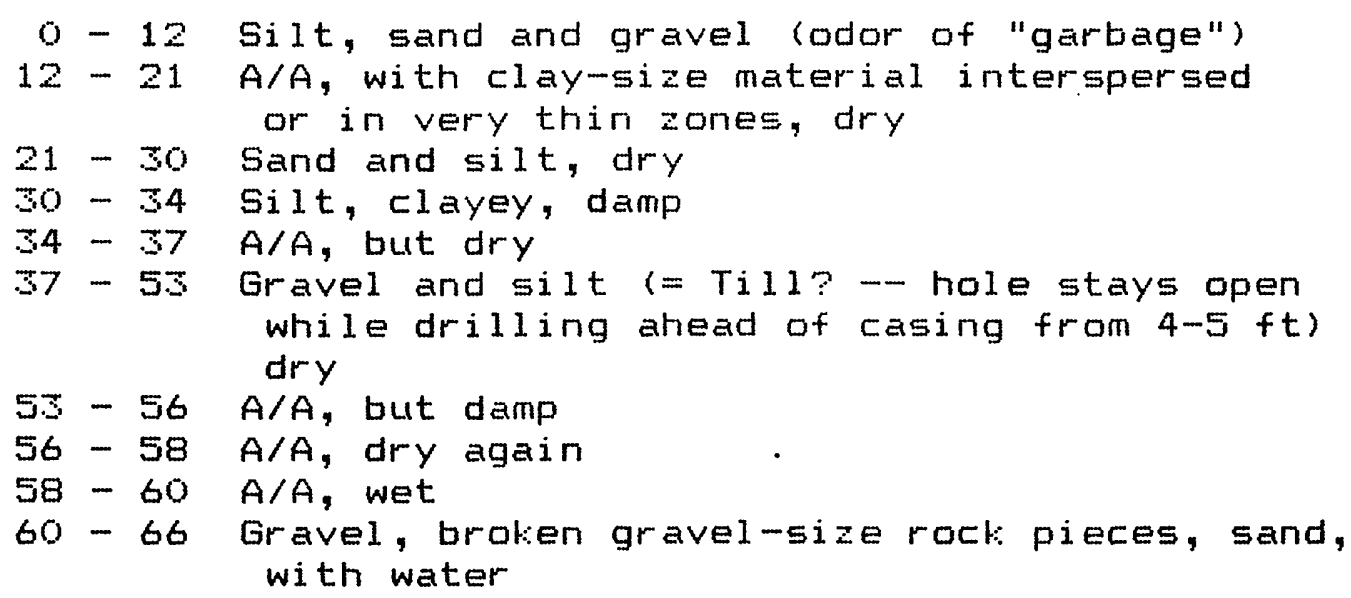


BIG LAKE SITE -- Mat-Su Landfills (cont)

Big Lake LF No. 3

[USGS ID $613235 \quad 149472801]$

Well depth - 37.5 feet

Water level -- 12 feet below 1 sd

Well finish - Dpen end

Well 109 --

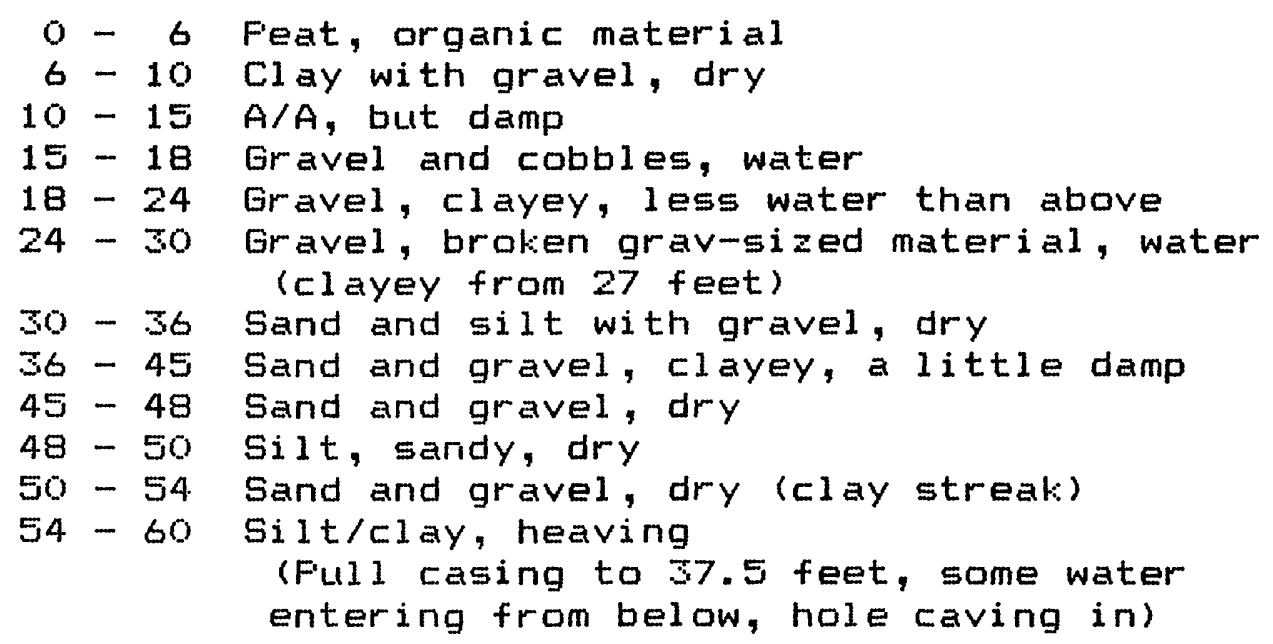


Leo A. Brueggeman

Star Rt. Box 2275

Wasilla, Alaska 99687
BLUE BEAR DRILLING
Telephone 892-6342

Mile 1, Big Lake Road

WATER WELL LOG

Property Owner $170 T=$ SLI BOLC:LiGiA

Location

Salitiry Fice

Date Completed

$8-28-81$

LF -5

Mailing Address

$B / G<a K C, A K$

Driller

Depth of Well \&5 $\mathrm{ft}$.

Casing Diameter $\sigma_{\text {in. }}$

Total Casing Installed

Static Water Level (measured from ground surface)

Well Yield GPM

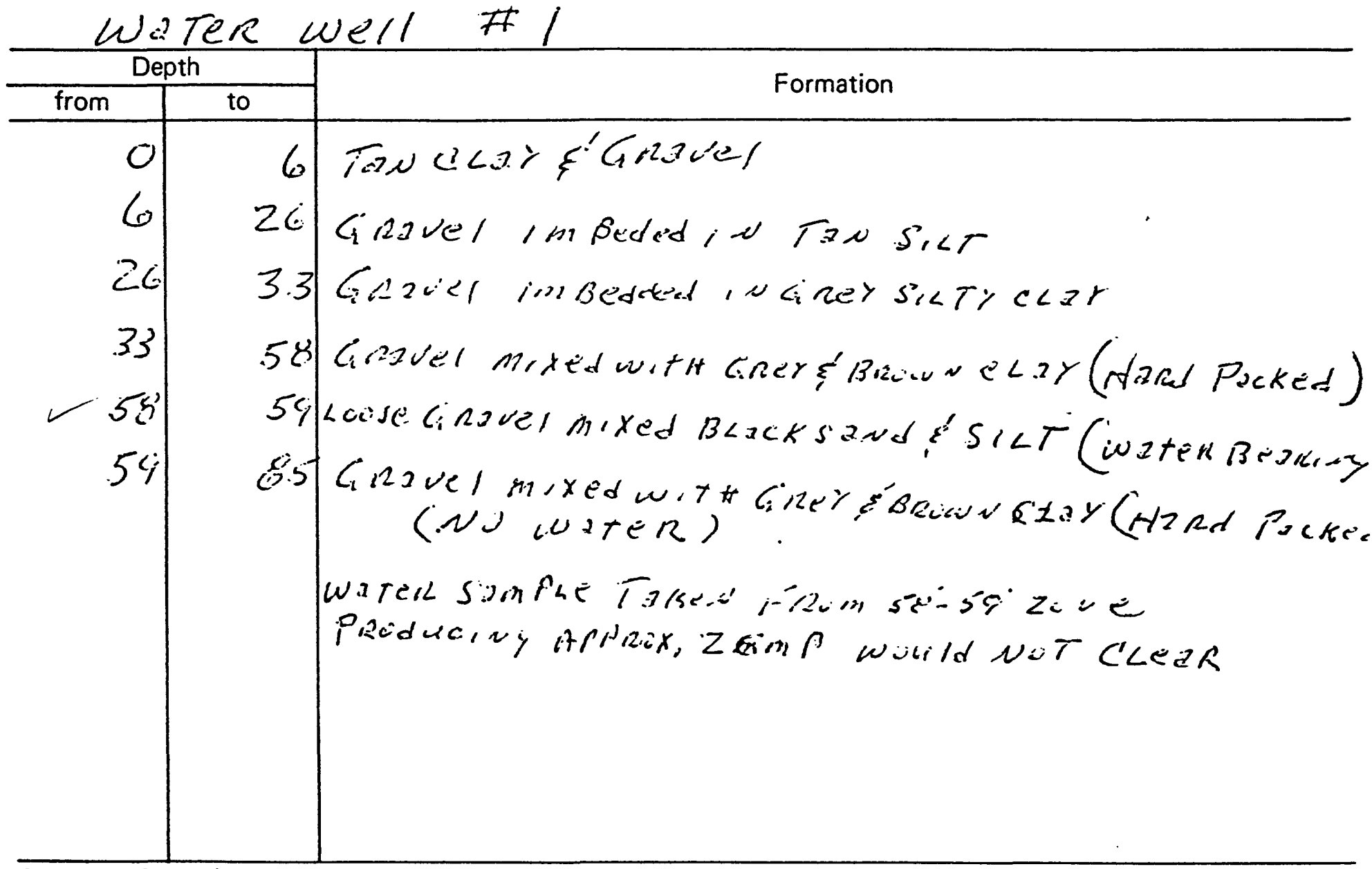


Leo A. Brueggeman

Star Rt. Box 2275

Wasilla, Alaska 99687
BLUE BEAR DRILLING

WATER WELL LOG
Telephone 892-6342

Mile 1, Big Lake Road Date Completed $8-2 \varepsilon^{\prime}-81$

Property Owner MUT-SU BoRouGH Location SON I TREY EIL

Mailing Address $B, y<.8 \Gamma \mathrm{e}, A K$

Driller

Depth of Well $70 \mathrm{ft} . \quad$ Casing Diameter 6 in. Total Casing Installed Static Water Level (measured from ground surface) 12 Well Yield 1.5 GP

Water Well \#2

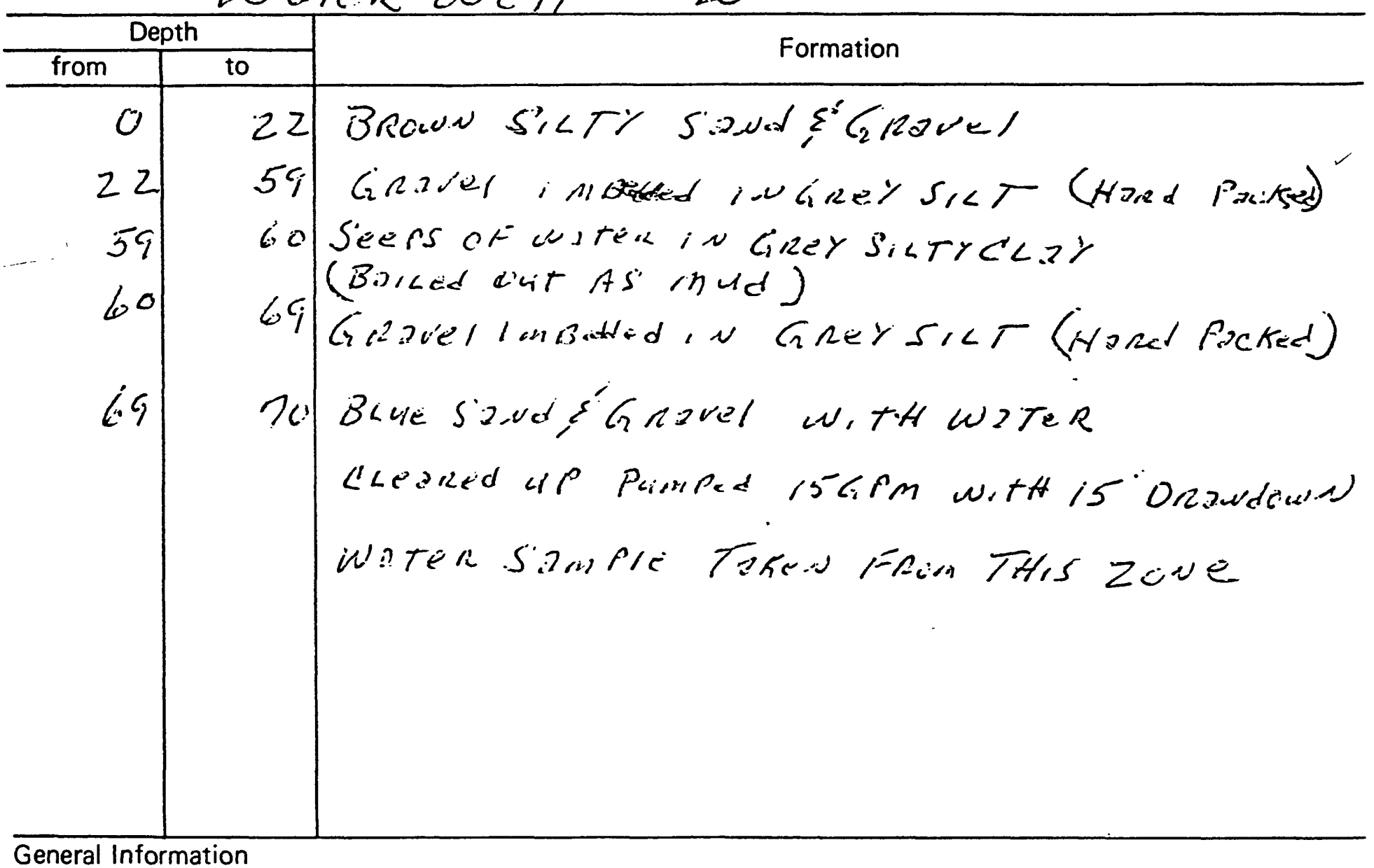

General Information 


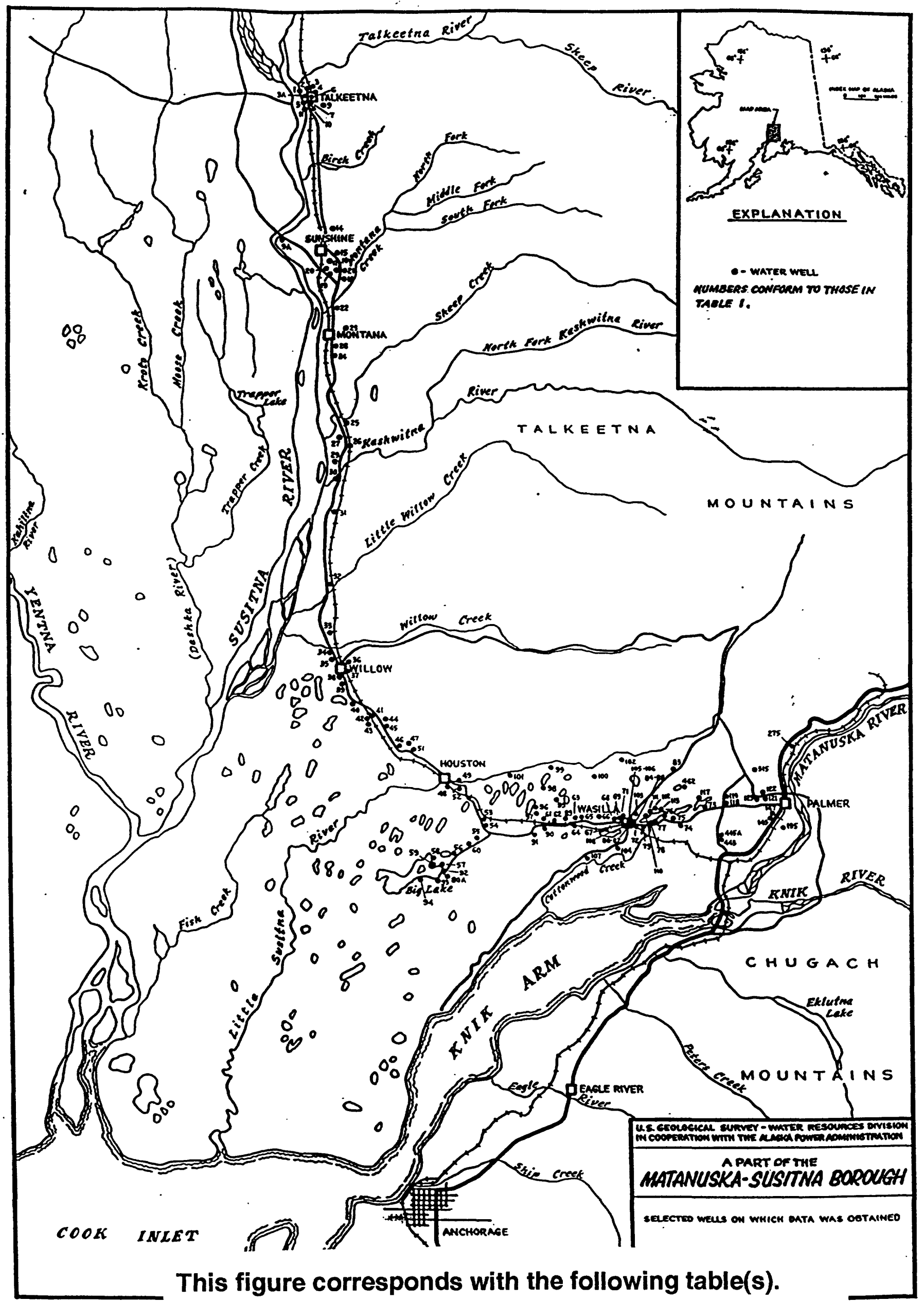




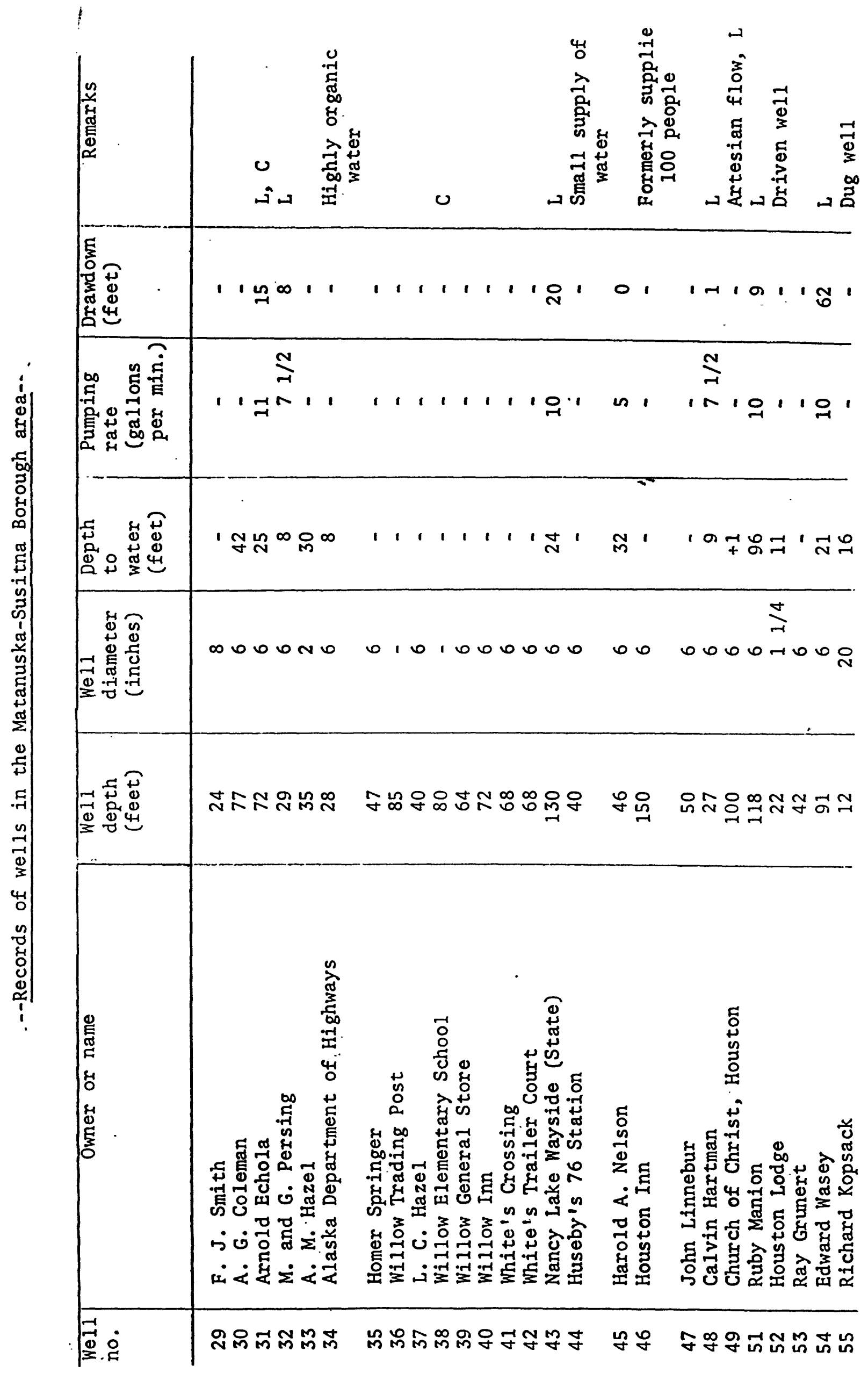




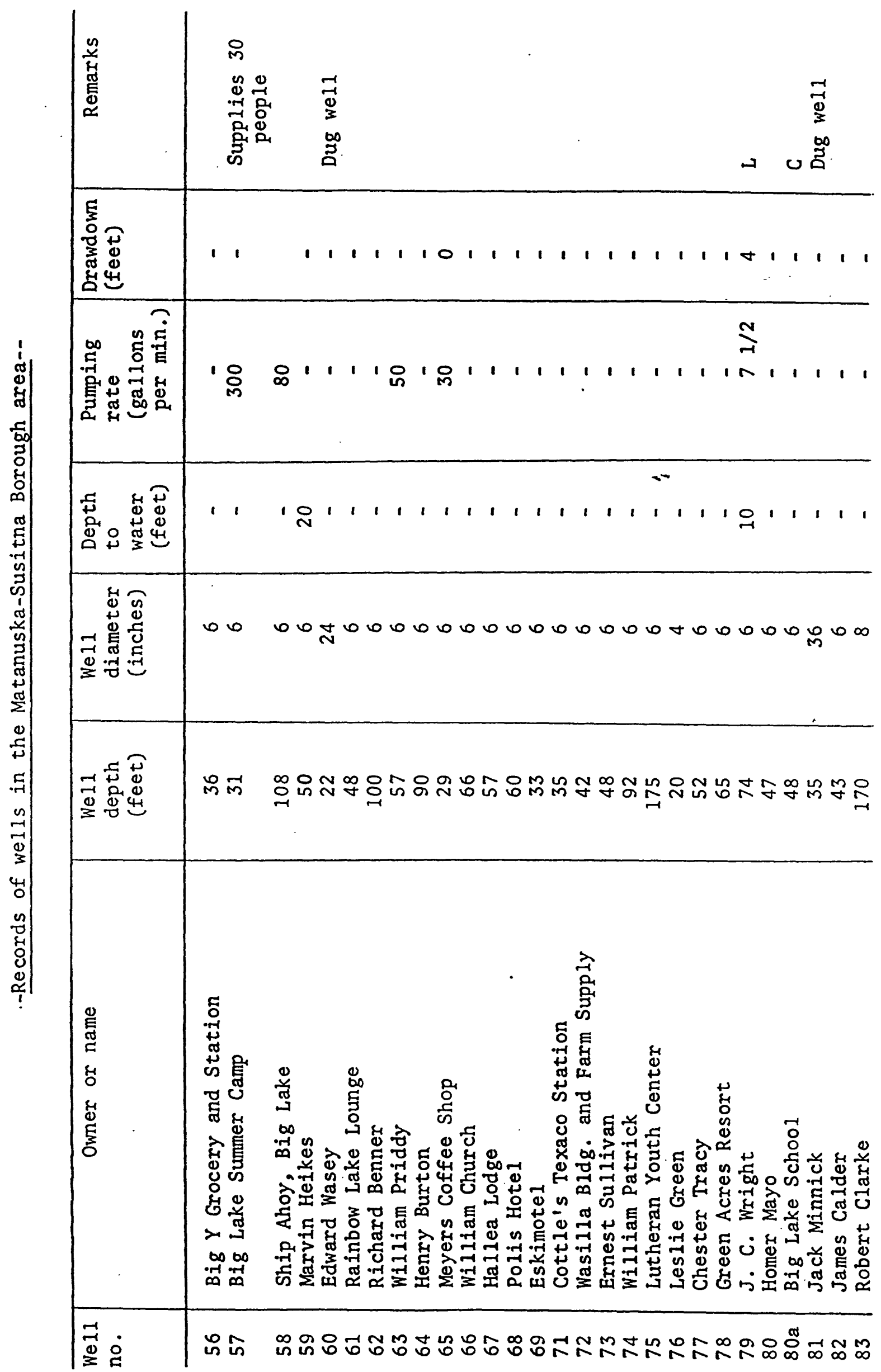




\begin{tabular}{|c|c|}
\hline 音 & ルルロ \\
\hline 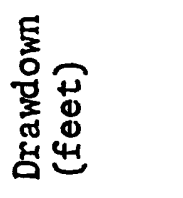 & 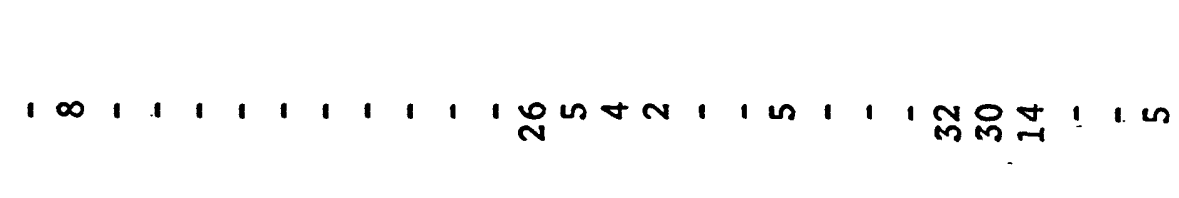 \\
\hline 点 & 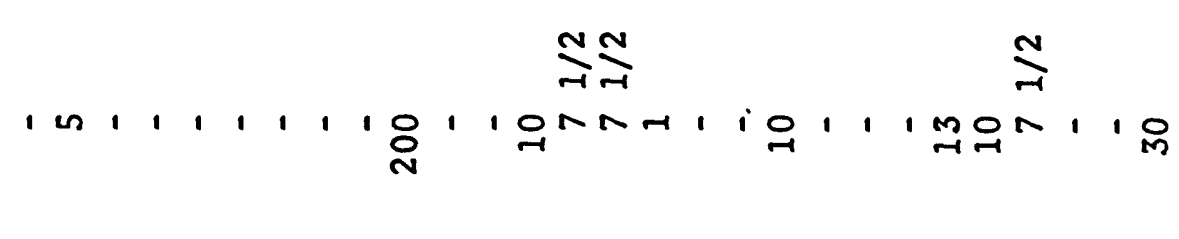 \\
\hline 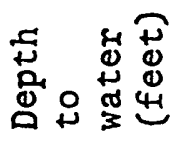 & 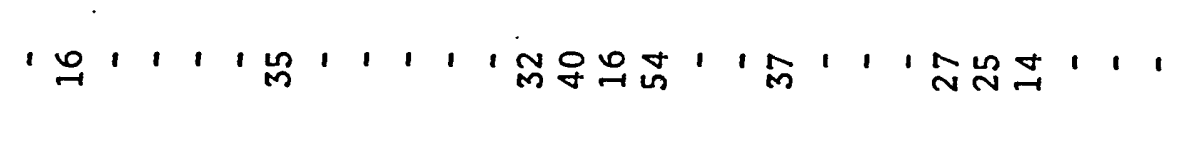 \\
\hline 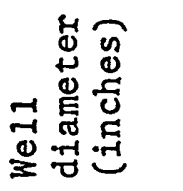 & 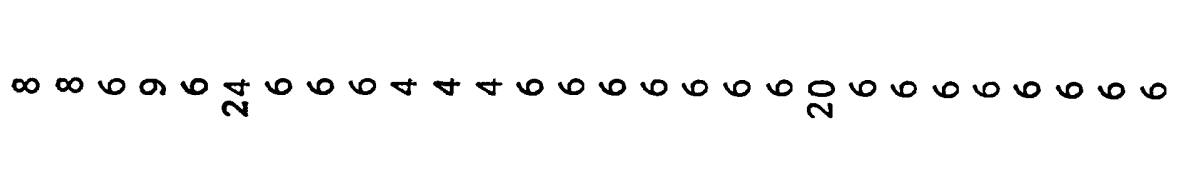 \\
\hline 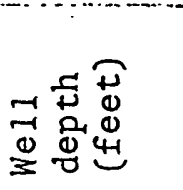 & 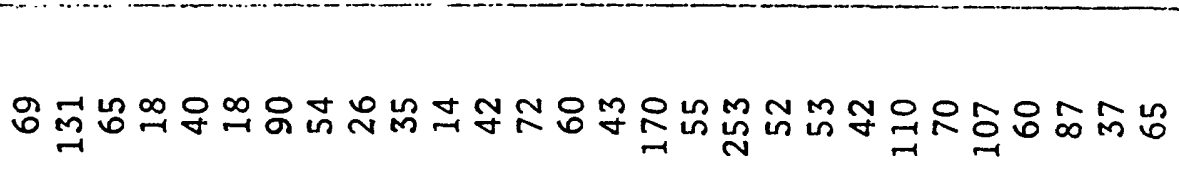 \\
\hline & 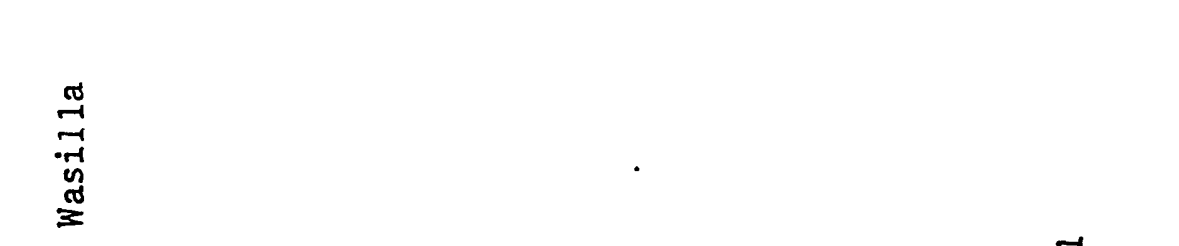 \\
\hline 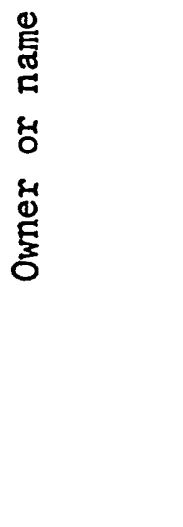 & 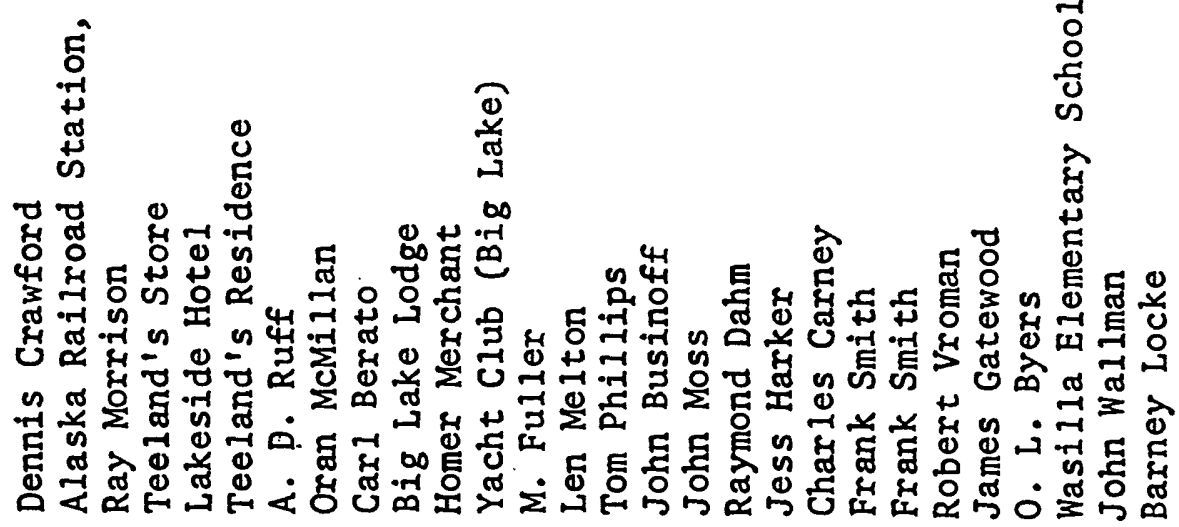 \\
\hline$\underset{\square}{\rightleftarrows} \dot{0}$ & せ্் \\
\hline
\end{tabular}




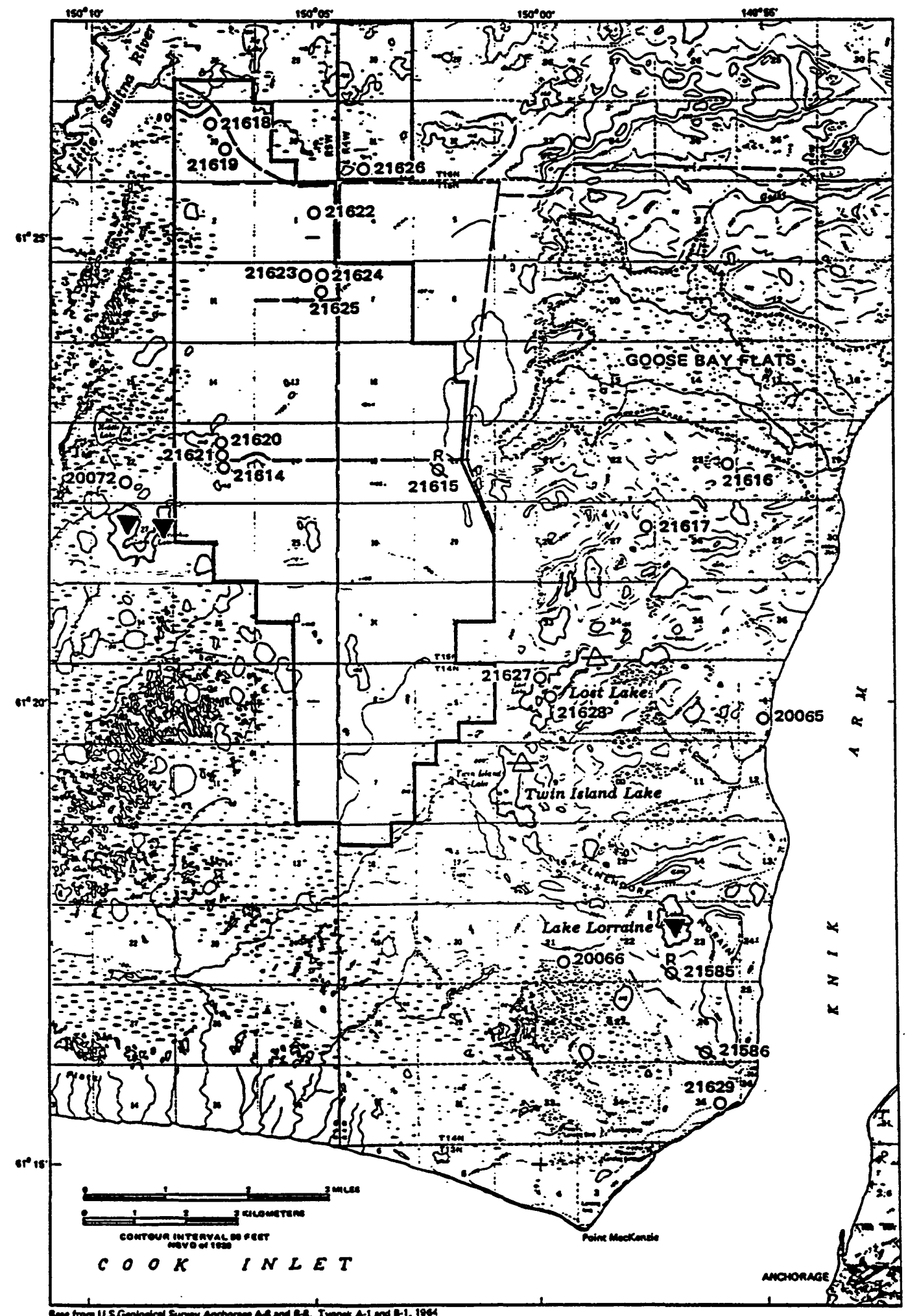

- Location of data-collection sites.

This figure corresponds with the following table(s). 
- Summary of well data

\begin{tabular}{|c|c|c|c|c|c|c|c|c|c|c|c|c|c|}
\hline $\begin{array}{l}\text { mell } \\
\text { eaber }\end{array}$ & amer er ave & erlller & $\begin{array}{c}\text { roar } \\
\text { comeleted }\end{array}$ & $\begin{array}{c}\text { Alel tuce } \\
\text { ift soove } \\
\text { sed } \\
\text { levell } \\
\end{array}$ & Depta & $\begin{array}{l}\text { Cepth of } \\
\text { epentegs } \\
\text { (fit) }\end{array}$ & Anulfor & $\begin{array}{c}\text { reld } \\
\text { (qulela) }\end{array}$ & 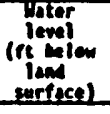 & Date & 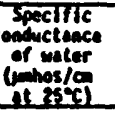 & $\begin{array}{l}\text { ate } \\
\text { ensures }\end{array}$ & Deants \\
\hline 20072 & calf ofl & Pean dersoy & 1969 & III & 200 & 200 & Conflaned & os & 3 & $01-01-c 9$ & $\cdots$ & - & $\cdots$ \\
\hline 20073 & Wart Gy & tean dersey & 197 & 70 & 298 & 298 & Conflined & 176 & 30 & Datanem & $\cdots$ & $\cdots$ & $\begin{array}{l}\text { Mater reperted oy ener to } \\
\text { be teo saity te driak. }\end{array}$ \\
\hline 21505 & Mutremetke-Susitan Sorough & $n-n$ & 1981 & 130 & $\because$ & $339-398$ & Confined & 310 & $\begin{array}{l}105 \\
104.82 \\
104.42 \\
104.45 \\
104.87 \\
104.39 \\
104.82 \\
105.51 \\
105.38 \\
105.28 \\
104.06\end{array}$ & $\begin{array}{l}03-10-01 \\
07-20-01 \\
09-15-01 \\
0-17-01 \\
12-03-81 \\
03-23-01 \\
05-19-82 \\
05-22-42 \\
02-27-92 \\
02-23-22 \\
02-23-03\end{array}$ & 675 & $20-15-01$ & 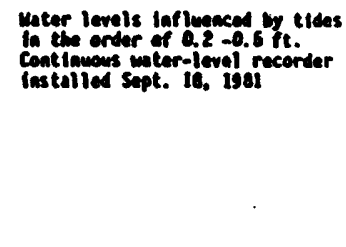 \\
\hline 21586 & Mutanuska-Sust tan Lorough & $n-n$ & 1981 & 152 & 358 & $312-323$ & Confiead & is & 142 & $03-03-81$ & 330 & $02-12-81$ & $\begin{array}{l}\text { Mater levels influeaced oy } \\
\text { ildes in the order of } 2 \text { ft. }\end{array}$ \\
\hline 21614 & usas test $m 11$ & $n-n$ & 1983 & 140 & 231 & 231 & confined & so & $\begin{array}{l}11.32 \\
11.10 \\
\text { II.14 }\end{array}$ & $\begin{array}{l}09-07-83 \\
0-17-83 \\
10-06-83\end{array}$ & 200 & $09-17-03$ & $\begin{array}{l}\text { Orllled to } 200 \text { it in Sept. } 1982 . \\
\text { deepened to } 231 \text { ft in Aug. 1983. }\end{array}$ \\
\hline 21615 & uses test mill & $n-n$ & 1982 & 185 & 310 & 310 & Confined & .1 & $\begin{array}{l}90.0 \\
90.8 \\
69.6 \\
90.11 \\
9.98 \\
8.81 \\
0.21 \\
9.11 \\
9.40\end{array}$ & $\begin{array}{l}10-12-82 \\
10-20-82 \\
02-10-83 \\
04-28-83 \\
06-16-83 \\
06-01-83 \\
00-89-83 \\
6-19-83 \\
10-06-83\end{array}$ &.$^{2100}$ & $10-12-12$ & $\begin{array}{l}\text { Cont Inuous mator level recorder } \\
\text { Installed Oct. 12, } 1982 \text {. }\end{array}$ \\
\hline 21616 & Solva faes & $n-4$ & 1982 & 65 & 3 & 73 & Wator table & 6 & $\begin{array}{l}60 \\
58.95 \\
5.03 \\
5.12\end{array}$ & $\begin{array}{l}07-12-82 \\
06-16-83 \\
00-01-83 \\
05-19-13\end{array}$ & & & i \\
\hline 21618 & Greg tell & $n-n$ & 1982 & 200 & 300 & 300 & Confined & 1 & $\begin{array}{l}141.22 \\
144.33 \\
141.21\end{array}$ & $\begin{array}{l}06-16-83 \\
00-01-83 \\
0-19-81\end{array}$ & & & $\therefore$ \\
\hline 21cis & Karea lea & $n-M$ & 1982 & 120 & 23 & 233 & Confleed & 200 & $\begin{array}{l}2 \\
4.550 \\
5.100\end{array}$ & $\begin{array}{l}07-29-02 \\
06-06-03 \\
09-19-01\end{array}$ & 350 & $6-24-62$ & \\
\hline 21619 & Karen lee & $n-n$ & 1982 & 120 & 50 & 50 & Mater table & s & $\begin{array}{l}20 \\
26.67 \\
27.11 \\
27.39\end{array}$ & $\begin{array}{l}07-23-02 \\
06-16-03 \\
0-01-03 \\
0-19-03\end{array}$ & 200 & $\cos -24-22$ & . \\
\hline 21620 & miloura Tucter & Mutea & 1983 & 125 & 206 & 206 & Confined & 150 & c & $06-01-03$ & 208 & $10-06-83$ & $\cdot$ \\
\hline 21621 & Milburn Tuckor & meaton & 1943 & 126 & 242 & 242 & Conflued & 300 & 8 & $06-01-83$ & 205 & $10-06-83$ & \\
\hline 21622 & Or. John danes & Durbin & 1923 & 145 & 6 & $\mathbf{c o}$ & Mater table & 10 & 25 & $06-10-81$ & 200 & $10-06-03$ & \\
\hline 21623 & MR farms 11 & Durbin & 1943 & 155 & co & $\omega$ & Mater table & 35 & 27 & 06-10-83 & 230 & $10-06-83$ & \\
\hline 21624 & MBR farms 12 & Durbin & 1943 & 155 & co & 160 & Weter table & 30. & 24 & $07-01-03$ & 210 & $10-06-83$ & \\
\hline 21625 & ma fand is & Durbin & 1983 & 150 & $\infty$ & $\mathbf{c o}$ & Mater teble & 30 & 24 & $07-01-83$ & $\cdots$ & -- & $\cdot$ \\
\hline 21626 & Sence Mright & noen & 1983 & 145 & 60 & 60 & Water table & $\because$ & $\cdots$ & $\cdots$ & 200 & $06-10-63$ & \\
\hline 21627 & Sack Cuthane & Pena dersey & 1983 & 130 & 256 & 256 & Conflined & 10 & 93 & $03-02-83$ & 1020 & $\cdots$ & Mater contaliss some gas. \\
\hline 21620 & Jerry Culhene & Pema versey & 1983 & 130 & 191 & 181 & Conftined & 30 & 80 & $02-11-83$ & 890 & $\cdots$ & Mater conta ins some gas. \\
\hline 21629 & Moy Syren & Syren Dros. & 1982 & 140 & 196 & 196 & Conflaned & 25 & 130 & $03-12-82$ & $\cdots$ & $\cdots$ & \\
\hline
\end{tabular}

- Mecently pumped 
Well 20072, Gulf 0i1, NW/ SW sec.22, T. 15 N., R.5 W. [Log by Penn Jersey]

$\begin{array}{ll}0 \text { to } 14 & \text { Muskeg } \\ 14 \text { to } 19 & \text { Sand (water) } \\ 19 \text { to } 40 & \text { Clay and clay } \\ 40 \text { to } 62 & \text { Sand and } \\ 62 \text { to } 168 & \text { Quicksand } \\ 168 \text { to } 194 & \text { Clay (water) } \\ 194 \text { to } 197 & \text { Sand (water) } \\ 197 \text { to } 200 & \text { Gravel (water) }\end{array}$

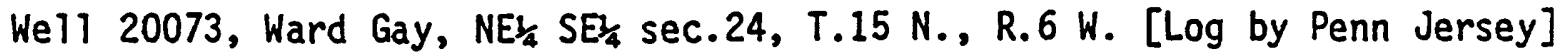

0 to 80

80 to 290

290 to 300
Sand

Sand and silt Gravel

We11 21585, Matanuska-Susitna Borough test well, SW/ SW/

[Log by USGS]

1 to 4

4 to 15

15 to 26

26 to 35

35 to 55

55 to 63

63 to 125

125 to 130

130 to 170

170 to 188

188 to 197

197 to 219

219 to 284

284 to 294

294. to 309

309 to 312

312 to 323

323 to 338

338 to 398
Silty sand and gravel

Silty sand and gravel (water)

silty sand (water)

Clay

Silty, sandy clay (damp)

Clay

Hardpan

Silty sand and gravel (water)

Sand and gravel (water)

Clay

Gravelly clay

Clayey, silty sandy gravel

Sand and gravel (water)

Sand (water)

Sand and gravel (water)

Gravelly sand (water)

silty sand (water)

Silty sand and gravel (water)

Sand and gravel (water) 


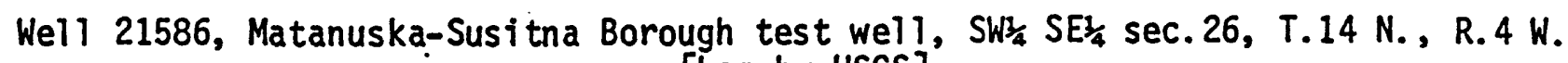
[Log by USGS]

1 to 8
8 to 18
18 to 23
23 to 31
31 to 106
106 to 116
116 to 156
156 to 166
166 to 185
185 to 198
198 to 203
203 to 219
219 to 225
225 to 238
238 to 328
328 to 339
339 to 349
349 to 351
351 to 352
352 to 358

Sand and gravel

Silty sand

Sand (water)

Clay, gray

Gravelily clay, gray

Clayey grave 1

Sil ty grave 1

Sand and gravel (water)

Hard dry silt

Sand and gravel (water)

Sand (water)

Silt, sand, and gravel

Sandy, sand and gravel (water)

Gravel (water)

Sand and gravel (water)

Sand, heaving (water)

Silty sand and gravel, heaving (water)

Silty gravel

Cemented sand and gravel

Clay, sticky

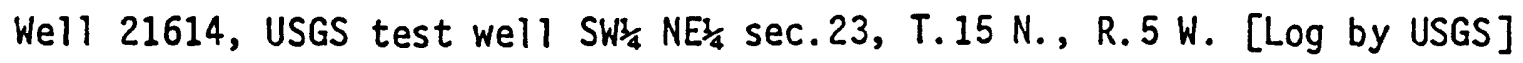

0 to 14

14 to 43

43 to 78

78 to 80

80 to 104

104 to 111

111 to 133

133 to 145

145 to 158

148 to 178

178 to 200

200 to 210

210 to 213

213 to 215

215 to 220

220 to 224

224 to 227

227 to 231
Sand and gravel

Sand and gravel (wet)

Clay, gray

Silty sand and gravel, gray

Silty sand, gray

Silty clay, gray

Sand, gray

Graveliy silt, gray

Sandy silt, gray

Gravelly silt, gray

Sandy silt, gray

Sandy silt, gray

Medium-fine sand, gray (water to $210 \mathrm{ft}$ )

Silty, fine sand, gray

Coarse to very-fine sand and wood fragments

Silty sand and wood fragments Gravelly silt, gray (yields virtually no water)

Sand and gravel (water) 
Depth below land surface in feet

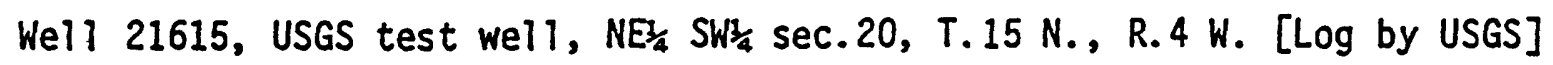

\section{Lithologic description}

Sand, gravel, and cobbles

Sand and gravel

Silt, brown

Sand (water)

Sil ty gravel

Silt, brown

Sand and gravel (water)

Silty sand and gravel

Sand and gravel (water)

Silt, gray

Clay, brownish gray

Clayey silt with thin sand lenses (water)

Gravelly silt

Clayey silt, gray

Sandy and clayey silt, gray

Silty gravelly clay, gray

Sand and gravel (water)

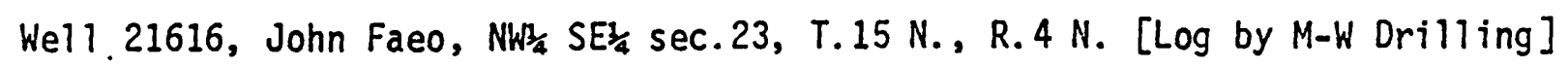

0 to 18

18 to 19

19 to 38

39 to 58

58 to 68

68 to 73
Topsoil

Very hard cobble gravel

Sand

Layered sand and gravel

Sand

Sand and gravel (water)

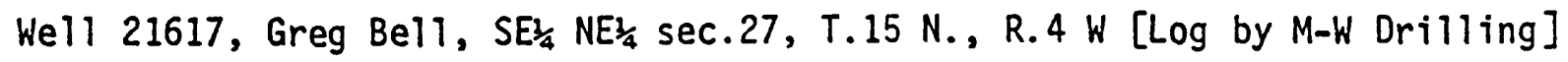

0 to 2
2 to 103
103 to 136
136 to 198
198 to 238
238 to 328
328 to 338
338 to 373
373 to 380
Topsoil

Sand, very fine

Silty clay

Clay

Silty clay

Clay

Clay - hardpan

Hardpan (tili)

Sand, very fine, heaving 


\section{Depth below land \\ surface in feet}

Lithologic description

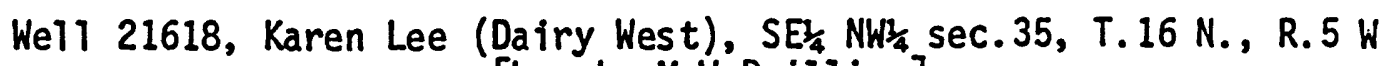
[Log by M-W Driliting]

0 to 26
26 to 30
30 to 58
58 to 78
78 to 86
86 to 98
98 to 104
104 to 106
106 to 200
200 to 206
206 to 219
219 to 239

Sandy gravel

Gravel (damp)

Sandy clay

Silty sand

Silty sand and clay

Clayish hardpan

Clay - hardpan

Hardpan and gravel (damp)

Clay

Sand, fine, gray

Silty sand and gravel

Gravel (water)

Wel1 21619, Karen Lee (Dairy West) NW/ $\mathrm{SE}_{\frac{1}{4}} \mathrm{Sec} .35$, T. 16 N., R. 5 W. [Log by M-W Driliing]

2 to 34

34 to 38

38 to 50

Sand and gravel

Sand (damp)

Sand and gravel (water)

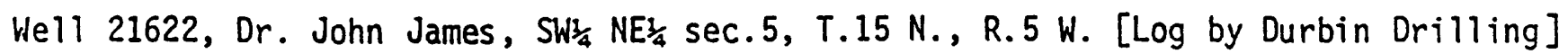

0 to 40

40 to 60

Sand and gravel

Sand and gravel (water)

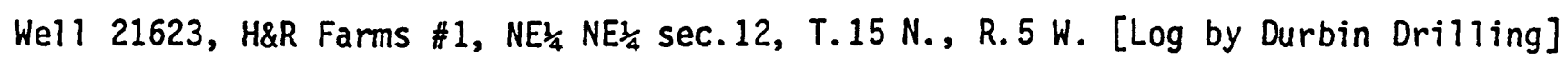
0 to 60

Sand and gravel

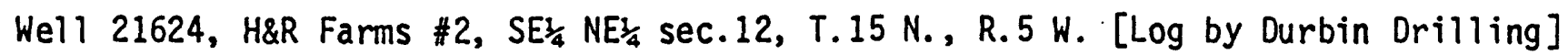
0 to 60 Sand and grave 1 


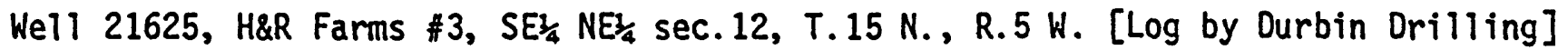

0 to 60

Sand and gravel

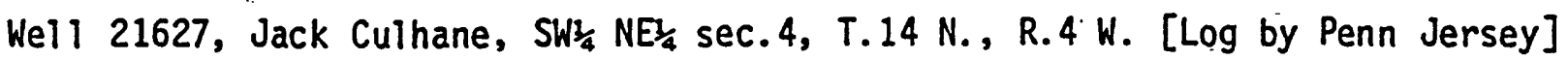

0 to 15
15 to 17
17 to 21
21 to 23
23 to 24
24 to 33
33 to 133
133 to 134
134 to 137
137 to 155
155 to 230
230 to 253
253 to 256

Topsoil

Clay and gravel

Sandy gravel (damp)

Clay

Boulder

Clay

Gray clay

Boulder

Clay and grave 1

Gray clay

Clay and gravel (damp)

Clay and gravel (dry)

Sand and gravel

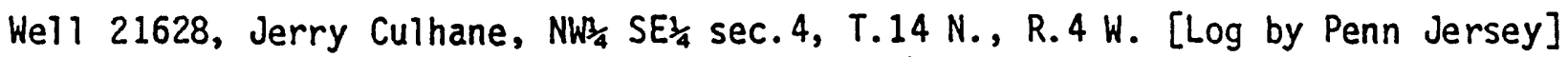

0 to 2

2 to 13

13 to 26

26 to 56

56 to 78

78 to 136

136 to 180

180 to 181
Topsoil

Clay and gravel (damp)

Gray clay and gravel

Clay and gravel (damp)

Clay and gravel

Clay

Clay and gravel

Sandy gravel

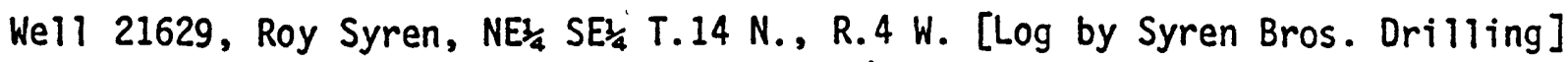

0 to 8

8 to 18

18 to 30

30 to 50

50 to 136

136 to 157

157 to 166

166 to 178

178 to 190

190 to 196
Gravel and sand

Sand and grave 1 (wet)

Gravel

Sandy gravel (wet)

Clay

Gravel and clay

Sand and gravel (damp)

Gray, silt and gravel (wet)

Sand, gravel, and silt (water)

Sand and gravel (water) 


\section{APPENDIX 2}

Water-quality data near Big Lake, Alaska 


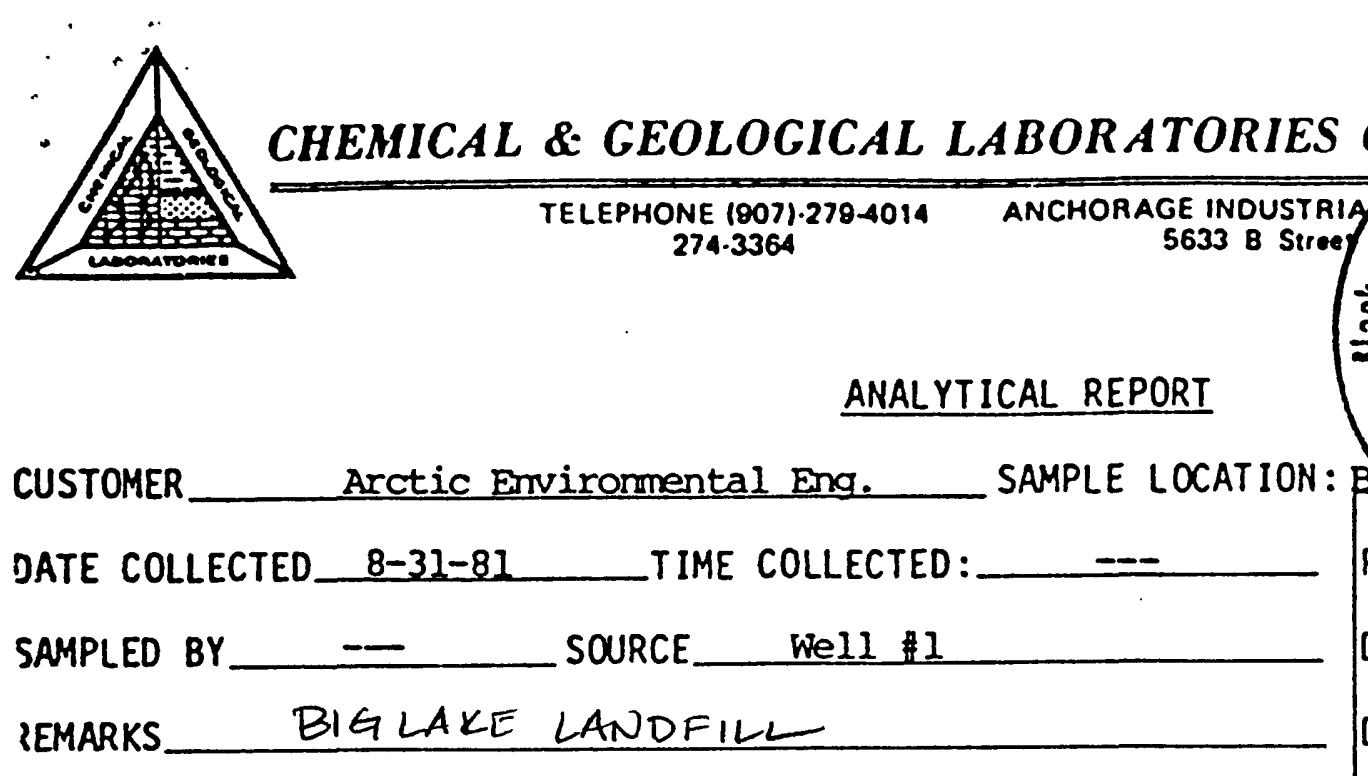

CUSTOMER_Arctic Environmental Eng.

DATE COLLECTED_ 8-31-81

TIME COLLECTED :

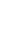

$\cdots$ FOR' ${ }^{\prime} A B$ OSE ONLY

DATE RECEIVED_ $8-31-8]$

DATE COMPLETED 2-8-8I

DATE REPORTED_9-9-81

SIGNED Archist. Shun

$$
\mathrm{mg} / \mathrm{l}
$$

JAg, Silver $<0.05$

JA1, Al uminum $\quad 0.10$

JAs, Arsenic < < 0.05

JAu,Gold $<0.05$

JB, Boron

$<0.05$

JBa, Barium $<0.05$

] J Bi, Bi smuth $<0.05$

.]Ca, Calcium

74

[]Cd, Cadmi um

$<0.01$

[]Co, Cobalt

$<0.05$

[ ]Cr,Chromium $<0.05$

[]Cu,Copper < <0.05

JFe, Iron

0.68

] $\mathrm{Hg}$, Mercury $<0.05$

.]K, Potassium

2.5

JMg, Magnesium 11

JMn, Manganese $\quad 0.38$

JMo, Molybdenum $<0.05$

JNa, Sodium

4.9

JNi,Nickel.

$<0.85$ $\underline{\mathrm{mg} / \mathrm{l}}$

[]P, Phosphorous $<0.05$

[] $\mathrm{Pb}$, Lead

$<0.05$

[]Pt,Platinum

$<0.05$

$<0.05$

[]Sb, Antimony

$<0.05$

[] Se, Sel enium

6.1

[]Si,silicon

$<0.10$

[ ]Sn, Tin

0.40

[]Sr, Strontium

$<0.05$

[]Ti,Titanium

$<1$

[]W, Tungsten

$<0.05$

[] V, Vanadium

0.12

[]Zn,Zinc

$<0.05$

[]Zr,Zirconium

[]Ammonia

Nitrogen-N

[]Kjedah]

Nitrogen-N

[]Nitrate-N

[]Nitrite-N.

[]Phosphorus

(Or tho) $-p$

[]Chloride

Q]Fluoride
[]Cyanide

[]Sulfate

[]Phenol

[]Total Dissoived Solids

[]Total Volatile Solids

[] Suspended Solids

[]Volatile Suspended Solids

[] Hardness as

$\mathrm{CaCO}_{3}$

[] Al kalinity as

$\mathrm{CaCO}_{3}$

[x sultide

$<0.002$

[X_COD

14

[]

[]

[]mmhos Conductivity

[]pH Units

[]Turbidity NTU

[]Color Units

[]T.Coliform/10anl

[]

[] 


\section{ANAL YTICAL REPORT}

STOMER Artic Enviromental Egrs.

TIME COLLECTED: 2015 HIS.

TE COLLECTED $8-20-81$

YPLED BY L. Brueggeman SOURCE Water Test Hole \#2

MARKS BIGLAKE
SAMPLE LOCATION:

FOR LAB USE ONLY
RECVD.BY GY LAB \#8632
DATE RECEIVED $8-21-81$
DATE COMPLETED $8-31-81$
OATE REPORTED $9-1-81$
SIGNEO A achie

$\underline{\mathrm{mg} / \mathrm{l}} \underline{\mathrm{mg} / \mathrm{l}}$

$\mathrm{Ag}$, Silver $\quad<0.05$

$A 1$, Aluminum $\quad 0.07$

As, Arsenic $\quad<0.05$

Au, Gold

$<0.05$

B, Boron

$<0.05$

Ba, Barium $<0.05$

Bi, Bismuth $<0.05$

Ca, Calcium 18

Cd, Cadmi um $<0.01$

Co, Cobalt

$<0.05$

$\mathrm{Cr}$, Chromium $<0.05$

ICu,Copper

$<0.05$

IFe, Iron

0.41

$\mathrm{Hg}$, Mercury

$<0.05$

K, Potassium

1.0

Mg, Magnesium

9.0

Mn, Manganese $<0.05$

Mo, Molybdenum $<0.05$

Na, Sodium

5.0

Ini.Nirkel

$<0.05$
[]P, Phosphorous $<0.05$

[] $\mathrm{Pb}$, Lead

$<0.05$

[ ] Pt, Platinum

$<0.05$

[]Sb, Antimony

$<0.05$

[] $\mathrm{Se}$, Sel enium

$<0.05$

[]Si, Silicon

4.1

[]Sn, Tin

$<0.10$

[]Sr, strontium

0.15

[]Ti,Titanium

$<0.05$

[]W, Tungsten

$<1$

[]$V$, Vanad ium

$<0.05$

[]Zn, Zinc

$<0.05$

[]2r,Zirconium

$<0.05$

[]Ammonia Nitrogen-N

[] Kjedahi

Nitrogen-N

[]Nitrate $-\mathrm{N}$

[]Nitrite-N

[]Phosphorus (Or tho) -P

[]Chloride

PIfluoride
[]Cyanide

[]Sulfa te

[]Phenol

[]Total Dissolved Solids

[]Total Volatile Solids

[]Suspended Solids

[]Volatile Suspended Solids

[] Hardness as $\mathrm{CaCO}_{3}$ 83

[] Al kalinity as

$\mathrm{CaCO}_{3}$

$\mathrm{mg} / \mathrm{l}$ 


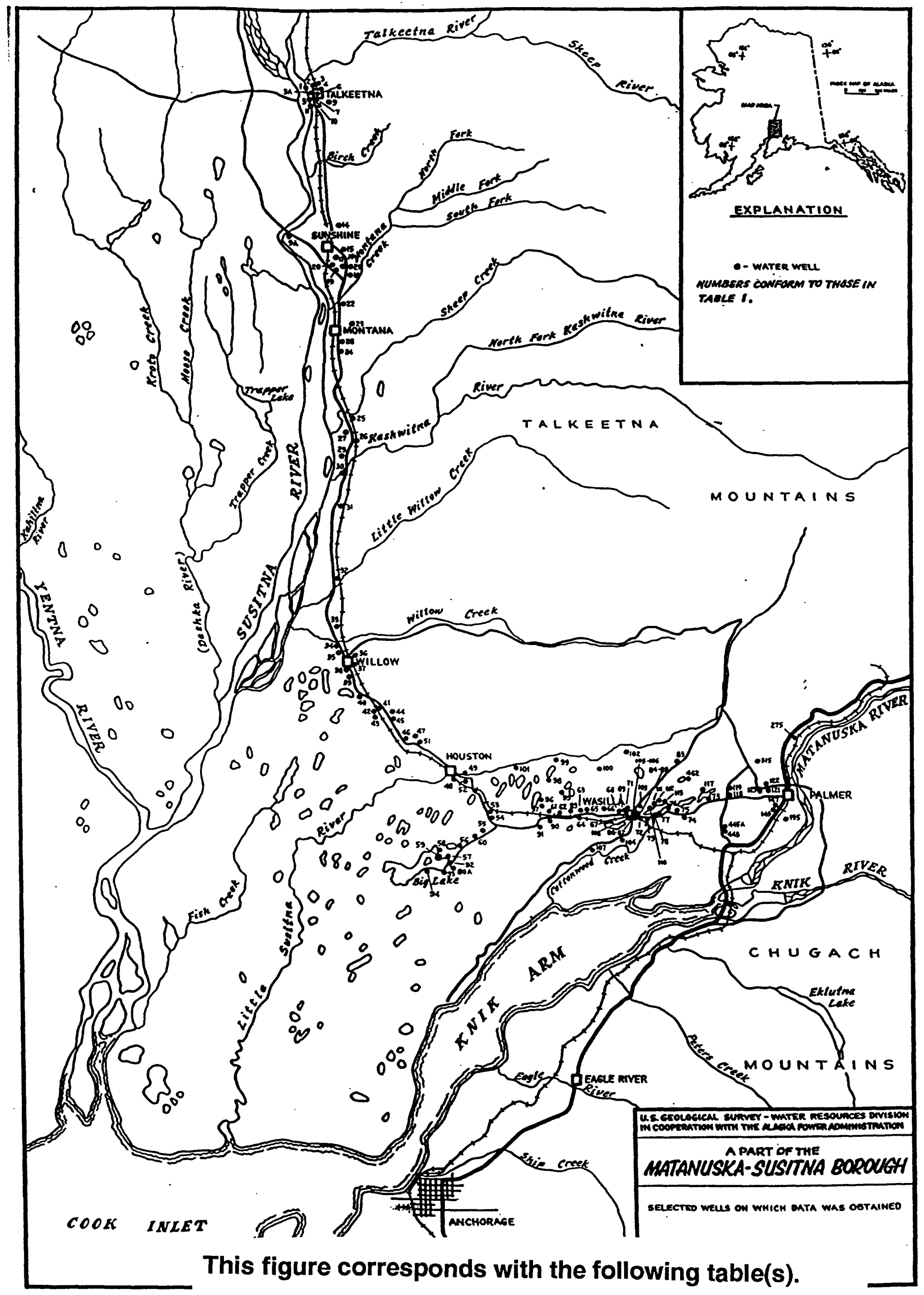




\begin{tabular}{|c|c|}
\hline$\frac{\mathrm{s}}{\mathrm{g}}$ & $\ldots \ldots \ldots \ldots$ \\
\hline$\underline{2}$ & 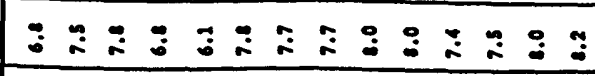 \\
\hline 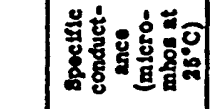 & 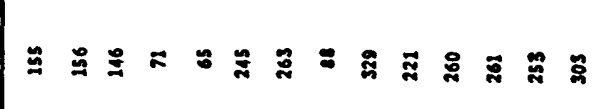 \\
\hline 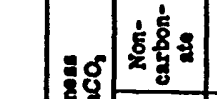 & $=\cdots \cdots,=\cdots$ \\
\hline 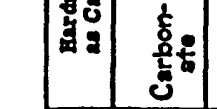 & 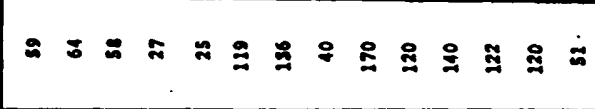 \\
\hline 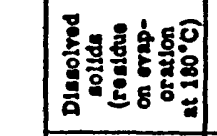 & $\therefore: a= \pm \equiv E=8$ \\
\hline 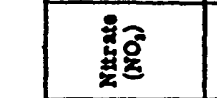 & : \\
\hline 咅 & 可 \\
\hline 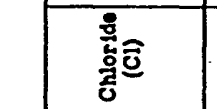 & 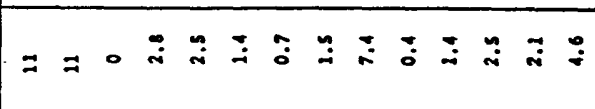 \\
\hline 产高 & 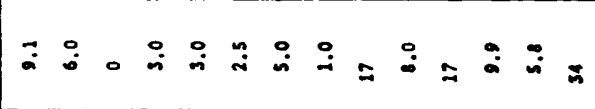 \\
\hline 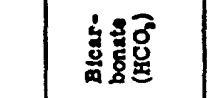 & 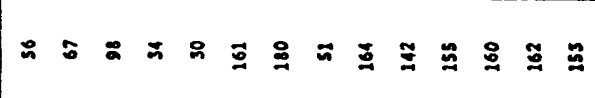 \\
\hline 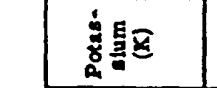 & 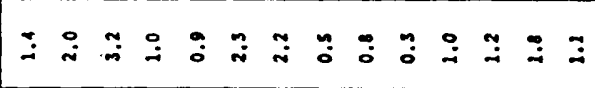 \\
\hline 嘓 & 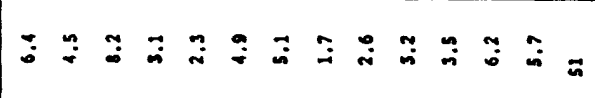 \\
\hline 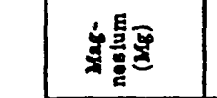 & 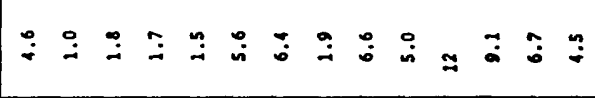 \\
\hline 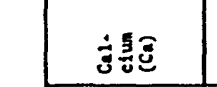 & 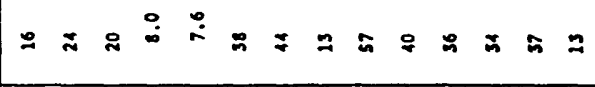 \\
\hline : & ỡ \\
\hline 然. & 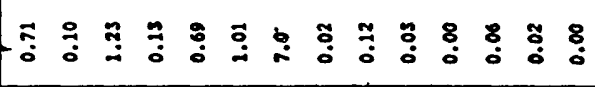 \\
\hline 害高" & 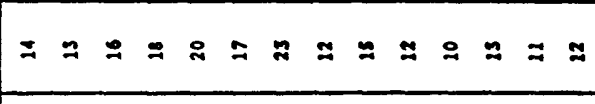 \\
\hline 竞 & 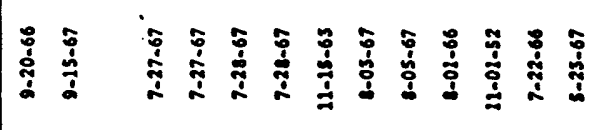 \\
\hline 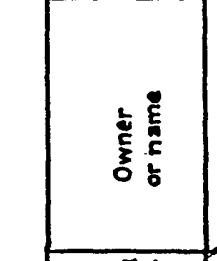 & 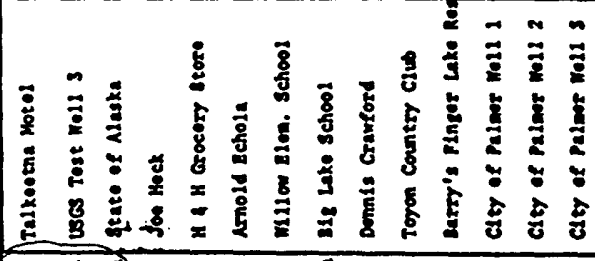 \\
\hline$z^{*}$ & 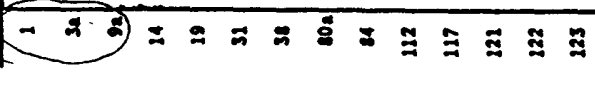 \\
\hline
\end{tabular}




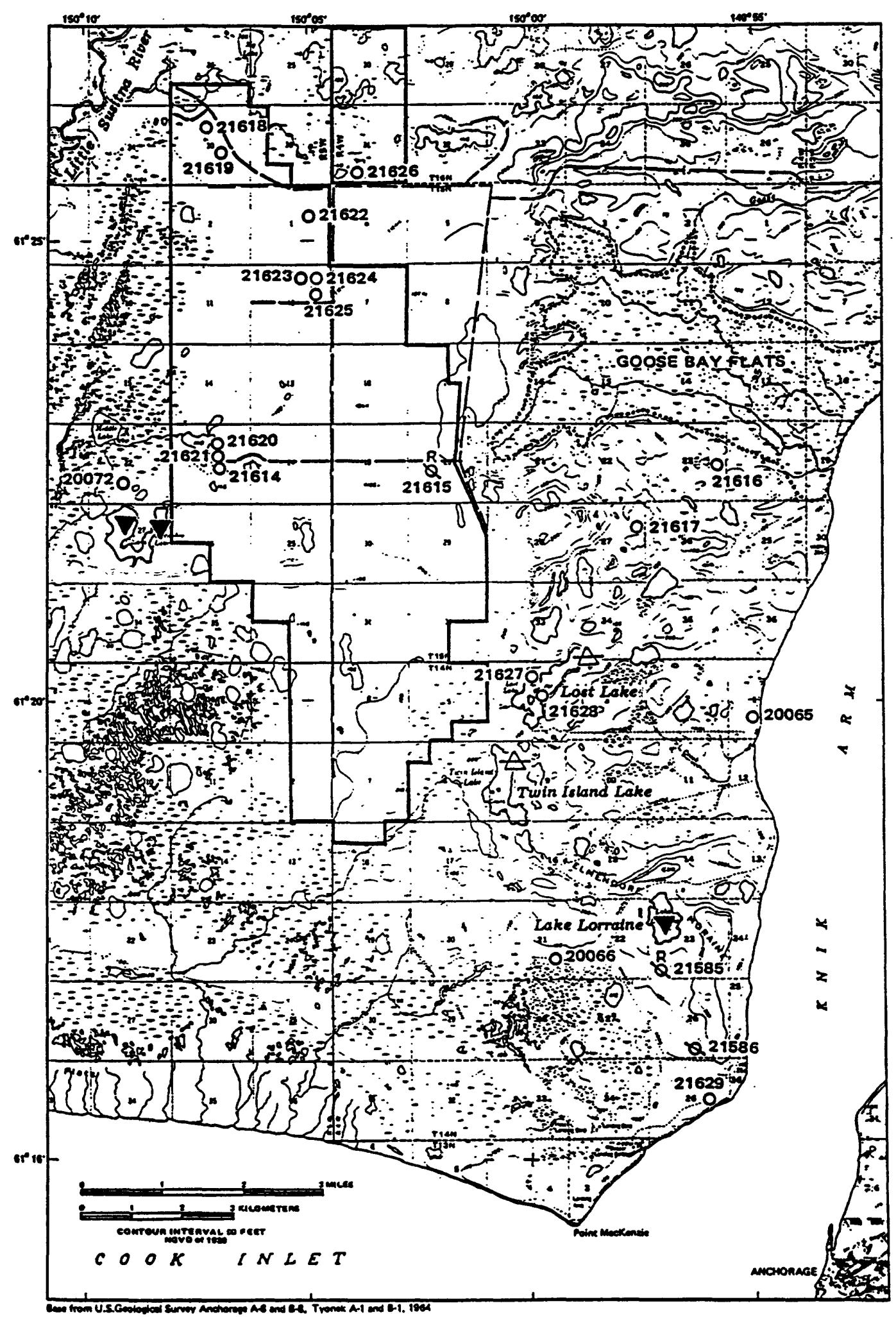

- Location of data-collection sites. 


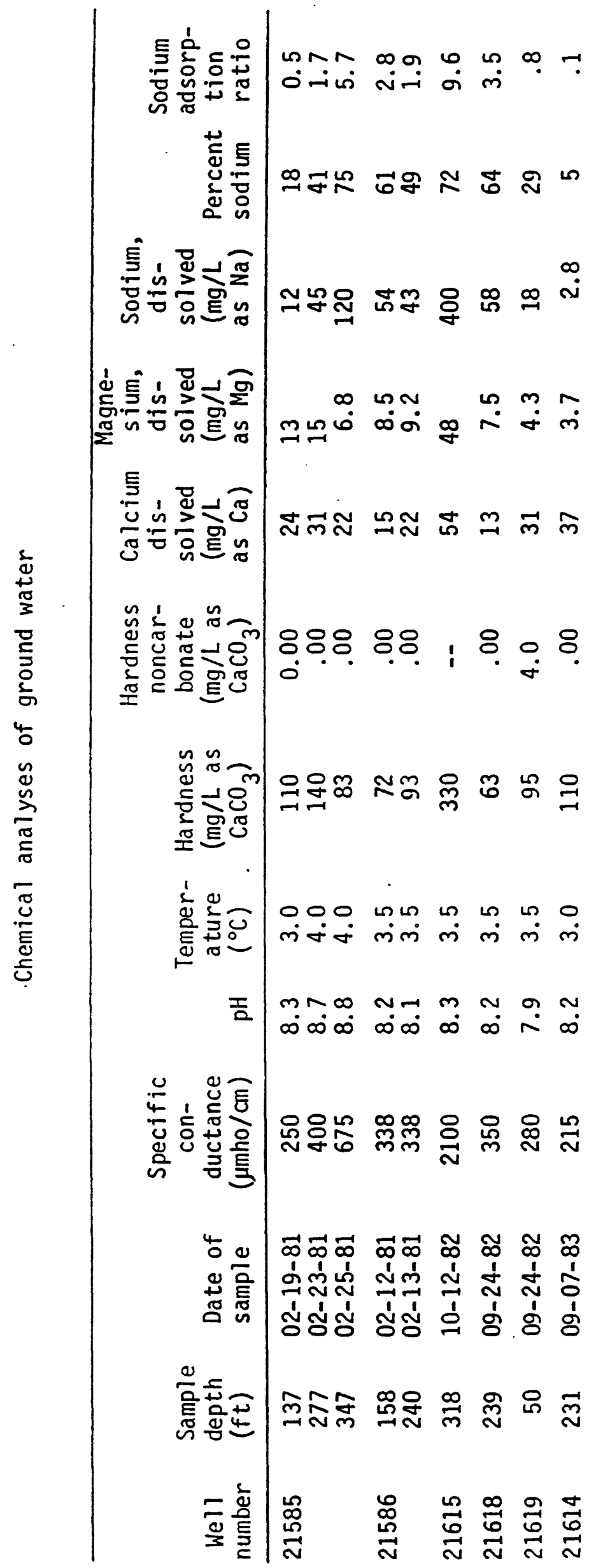

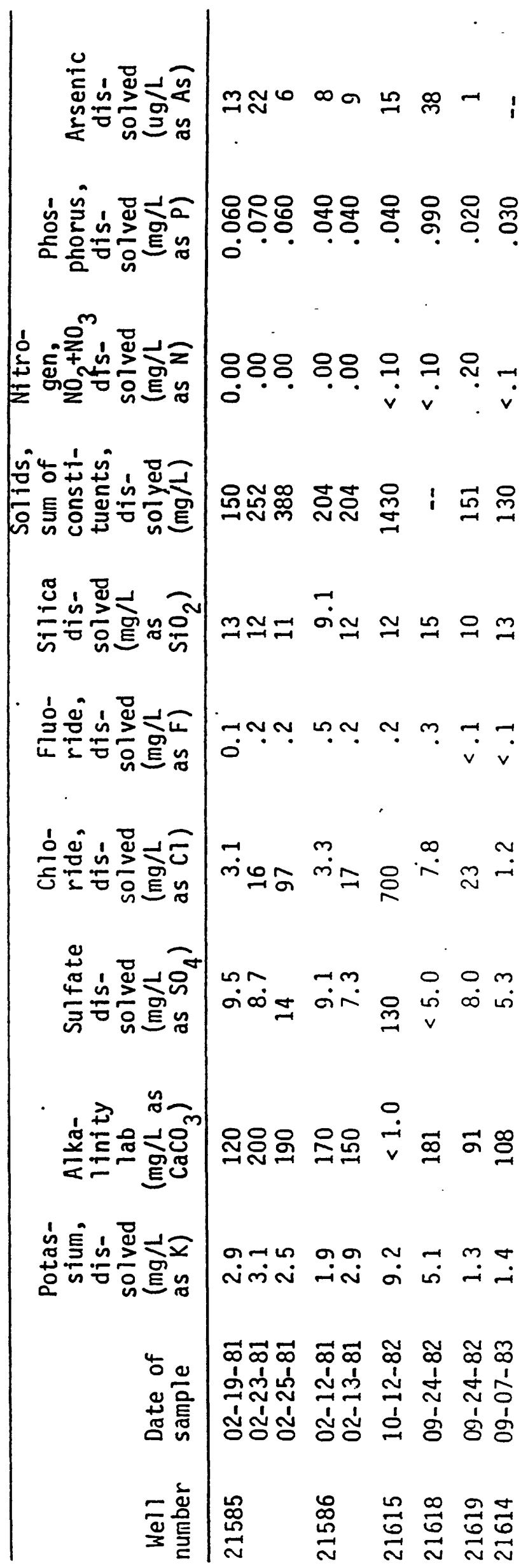


-Contínued

\begin{tabular}{|c|c|c|c|c|}
\hline $\begin{array}{c}\text { Well } \\
\text { number }\end{array}$ & $\begin{array}{l}\text { Date of } \\
\text { sample }\end{array}$ & $\begin{array}{l}\text { Boron } \\
\text { dis- } \\
\text { solved } \\
(\mu \mathrm{g} / \mathrm{L} \\
\text { as B) }\end{array}$ & $\begin{array}{c}\text { Iron, } \\
\text { dis- } \\
\text { solved } \\
\text { ( } \mu \mathrm{g} / \mathrm{L} \\
\mathrm{as} \mathrm{Fe})\end{array}$ & $\begin{array}{l}\text { Manga- } \\
\text { nese, } \\
\text { dis- } \\
\text { solved } \\
(\mu g / L \\
\text { as Mn) } \\
\end{array}$ \\
\hline $\begin{array}{l}21585 \\
21586 \\
21615\end{array}$ & $\begin{array}{l}02-19-81 \\
02-23-81 \\
02-25-81 \\
02-12-81 \\
02-13-81 \\
10-12-82\end{array}$ & $\begin{array}{r}90 \\
170 \\
200 \\
410 \\
160 \\
130\end{array}$ & $\begin{array}{r}30 \\
40 \\
60 \\
20 \\
40 \\
560\end{array}$ & $\begin{array}{r}100 \\
90 \\
60 \\
40 \\
80 \\
170\end{array}$ \\
\hline 21618 & $09-24-82$ & 200 & 100 & 76 \\
\hline 21619 & $09-24-82$ & 30 & 14 & 6 \\
\hline 21614 & $09-07-83$ & - & 130 & 94 \\
\hline
\end{tabular}


Records of Wells

-Chemical analyses of ground wator from the Matanuska Valley agricultural area, Alaska1

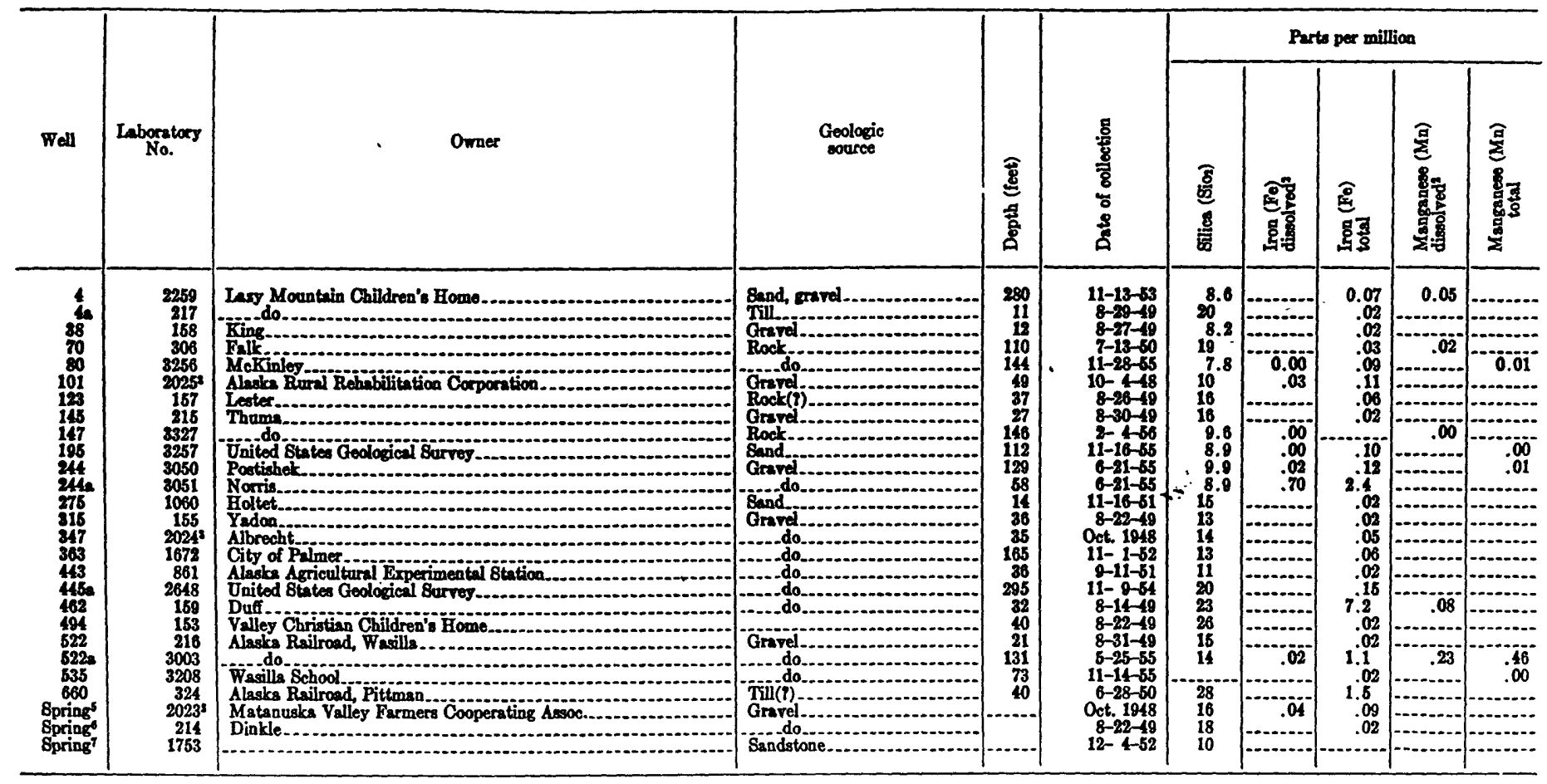

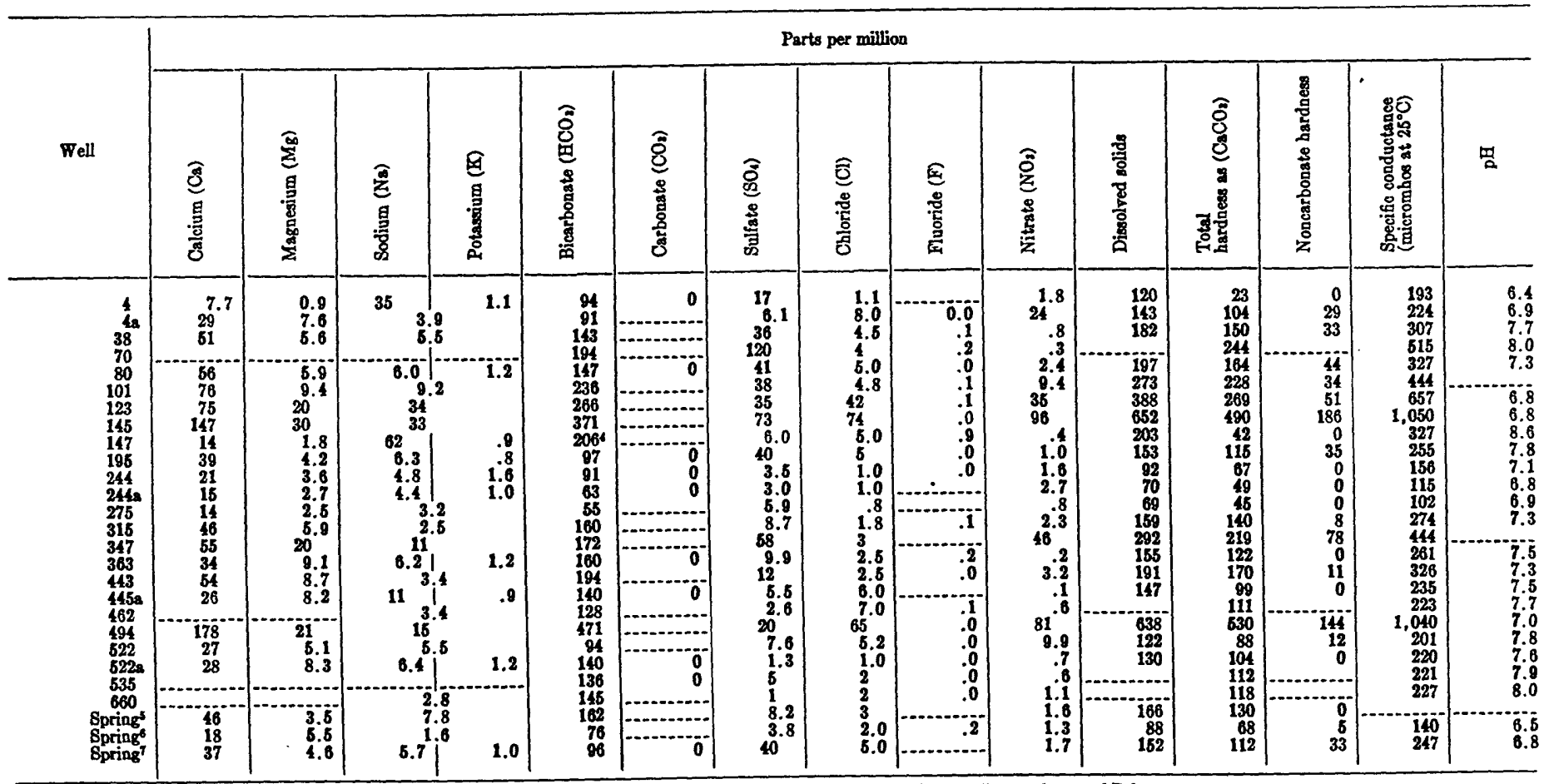

Inalyzes by Branch of Quality of Water, U.S. Geological Burvoy.

Brasil Spring, 3 miles northwest of Palmer.

'Salt Lake City laboratory $\mathrm{NO}^{\mathrm{O}}$. 